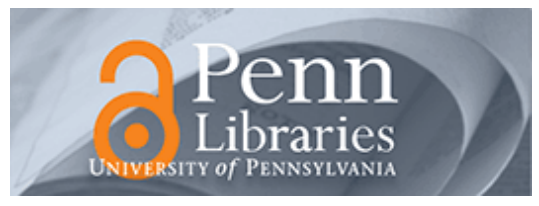

University of Pennsylvania

ScholarlyCommons

Statistics Papers

Wharton Faculty Research

1994

\title{
Optimal Stopping Rules and Maximal Inequalities for Bessel Processes
}

\author{
L. E. Dubins
}

Larry A. Shepp

University of Pennsylvania

A. N. Shiryaev

Follow this and additional works at: https://repository.upenn.edu/statistics_papers

Part of the Probability Commons

\section{Recommended Citation}

Dubins, L. E., Shepp, L. A., \& Shiryaev, A. N. (1994). Optimal Stopping Rules and Maximal Inequalities for Bessel Processes. Theory of Probability \& Its Applications, 38 (2), 226-261. http://dx.doi.org/10.1137/ 1138024

This paper is posted at ScholarlyCommons. https://repository.upenn.edu/statistics_papers/403

For more information, please contact repository@pobox.upenn.edu. 


\title{
Optimal Stopping Rules and Maximal Inequalities for Bessel Processes
}

\author{
Abstract \\ We consider, for Bessel processes $X \in$ Bes $^{\alpha}$ with arbitrary order (dimension) $\alpha \in \mathrm{R}$, the problem of the \\ optimal stopping (1.4) for which the gain is determined by the value of the maximum of the process $X$ and \\ the cost which is proportional to the duration of the observation time. We give a description of the optimal \\ stopping rule structure (Theorem 1 ) and the price (Theorem 2). These results are used for the proof of \\ maximal inequalities of the type \\ $E \max X_{r r} \leq r \leq \gamma(\alpha)$ is a constant depending on the dimension (order) $\alpha$. It is shown that $\gamma(\alpha) \sim \sqrt{ } \alpha$ at $\alpha \rightarrow$ \\ $\infty$.

\section{Keywords} \\ Bessel processes, optimal stopping rules, maximal inequalities, moving boundary problem for parabolic \\ equations (Stephan problem), local martingales, semimartingales, Dirichlet processes, local time, \\ processes with reflection, Brownian motion with drift and reflection

\section{Disciplines} \\ Probability | Statistics and Probability
}




\title{
OPTIMAL STOPPING RULES AND MAXIMAL INEQUALITIES FOR BESSEL PROCESSES*
}

\author{
L. E. DUBINS ${ }^{\dagger}$, L. A. SHEPP ${ }^{\ddagger}$, AND A. N. SHIRYAEVף
}

(Translated by N. A. Tolstoganova)

\begin{abstract}
We consider, for Bessel processes $X \in \operatorname{Bes}^{\alpha}(x)$ with arbitrary order (dimension) $\alpha \in \mathbf{R}$, the problem of the optimal stopping (1.4) for which the gain is determined by the value of the maximum of the process $X$ and the cost which is proportional to the duration of the observation time. We give a description of the optimal stopping rule structure (Theorem 1) and the price (Theorem 2). These results are used for the proof of maximal inequalities of the type

$$
\mathbf{E} \max _{r \leq \tau} X_{r} \leq \gamma(\alpha) \sqrt{\mathbf{E} \tau}
$$

where $X \in \operatorname{Bes}^{\alpha}(0), \tau$ is arbitrary stopping time, $\gamma(\alpha)$ is a constant depending on the dimension (order) $\alpha$. It is shown that $\gamma(\alpha) \sim \sqrt{\alpha}$ at $\alpha \rightarrow \infty$.

Key words. Bessel processes, optimal stopping rules, maximal inequalities, moving boundary problem for parabolic equations (Stephan problem), local martingales, semimartingales, Dirichlet processes, local time, processes with reflection, Brownian motion with drift and reflection
\end{abstract}

\section{Formulation of the problem. Main results}

1. A continuous non-negative Markov stochastic process $X=\left(X_{t}(x)\right)_{t \geq 0}, x \geq 0$, given on some filtered probability space $\left(\Omega, \mathcal{F},\left(\mathcal{F}_{t}\right)_{t \geq 0}, \mathbf{P}\right)$ is called a Bessel process of dimension (order) $\alpha \in \mathbf{R}\left(X \in \operatorname{Bes}^{\alpha}(x)\right)$ if $X_{0}(x)=x$, its infinitesimal operator is

$$
L=\frac{1}{2}\left(\frac{\alpha-1}{x} \frac{d}{d x}+\frac{d^{2}}{d x^{2}}\right)
$$

and the boundary point $x=\{0\}$ is a trap if $\alpha \leq 0$, a reflecting boundary if $0<\alpha<2$, and an entrance boundary if $\alpha \geq 2$.

(For a detailed study of Bessel processes $X \in \operatorname{Bes}^{\alpha}(x)$ see in [20], [13], [14], [17], [29] and later in $\S 2$.)

In case $\alpha \leq 0$ and $x=0$, the process $X_{t}(0) \equiv 0$ at $t \geq 0$.

In case $\alpha=1$, the process $X=\left(X_{t}(x)\right)_{t \geq 0}$ may be realized as the process $|B+x|=\left(\left|B_{t}+x\right|\right)_{t \geq 0}$, where $B=\left(B_{t}\right)_{t \geq 0}$ is standard $\left(B_{0}=0, \mathbf{E}\left(B_{t}-B_{s}\right)=\right.$ $\left.0, \mathbf{E}\left(B_{t}-B_{s}\right)^{2}=t-s, t \geq s \geq 0\right)$ Brownian motion.

In case $\alpha$ is an integer, $\alpha=d=2,3, \ldots$, the process $X \in \operatorname{Bes}^{d}(x)$ may be realized as a radial part of $d$-dimensional Brownian motion $B(a)=\left(B^{1}\left(a_{1}\right), \ldots, B^{d}\left(a_{d}\right)\right)$, i.e.,

$$
X_{t}(x)=\left[\sum_{i=1}^{d}\left(B^{i}\left(a_{i}\right)_{t}\right)^{2}\right]^{1 / 2}
$$

*Received by the editors October 2, 1992. The authors started the joint work over the given problems considered in the article in May-June 1992 in Bell Labs to which L. Dubins and A. Shiryaev were invited by L. Shepp. The main results stated in this paper were obtained in the same period.

†Department of Mathematics, University of California, Berkeley, California 94720.

‡AT\&T Bell Laboratories, Murray Hill, New Jersey 07974.

ISteklov Mathematical Institute, RAN, Vavilov str. 42, Moscow, Russia. 
where the vector $a=\left(a_{1}, \ldots, a_{d}\right)$ is such that its norm $|a|=x\left(|a|^{2}=\sum_{i=1}^{d} a_{i}^{2}\right)$ and $B^{1}\left(a_{1}\right), \ldots, B^{d}\left(a_{d}\right)$ are independent Brownian motions, exiting from the points $a_{1}, \ldots, a_{d}$, respectively.

2. Let $X \in \operatorname{Bes}^{\alpha}(x)$ and let $S=\left(S_{t}(x, s)\right)_{t \geq 0}$ be the stochastic process given by the formula

$$
S_{t}(x, s)=s \vee \max _{r \leq t} X_{r}(x),
$$

where $0 \leq x \leq s<\infty, a \vee b=\max (a, b)$.

Let us define for the Markov process $(X, S)$ under consideration the "value"

$$
V_{*}^{\alpha}(x, s)=\sup _{\tau} \mathbf{E}\left[S_{\tau}(x, s)-c \tau\right]
$$

where $c$ is some positive constant (the cost for the unit of observation) and the sup is taken over the class of all stopping times $\tau$ (i.e., the finite Markov time with respect to $\left.\left(\mathcal{F}_{t}\right)_{t \geq 0}\right)$.

The purpose of this paper is to study the problem of existence of optimal stopping times $\tau_{*}$ (for which $\mathbf{E}\left[S_{\tau_{*}}(x, s)-c \tau\right]=V_{*}^{\alpha}(x, s)$ for all $s \geq x \geq 0$ ), their structure and the structure of the value $V_{*}^{\alpha}(x, s)$ as a function of $x, s, \alpha$ (as well as that of "the cost" $c>0$ ). One of the main results of the work is the following.

Theorem 1. Assume $X \in \operatorname{Bes}^{\alpha}(x)$, where the dimensionality $\alpha \in R$ and $c>0$. The optimal stopping time $\tau_{*}$ (in problem (1.4)) exists and has the following form:

$$
\tau_{*}=\inf \left\{t \geq 0:\left(X_{t}, S_{t}\right) \in D_{*}\right\},
$$

with $X_{t}=X_{t}(x), S_{t}=S_{t}(x, s)$ and "stopping domain"

$$
D_{*}=\left\{(x, s): s_{*} \leq s, \quad x \leq g_{*}(s)\right\},
$$

where $g_{*}=g_{*}(s)$ is the non-negative (and unique) solution of the equation

$$
\frac{2 c}{\alpha-2} g^{\prime} g\left[1-\left(\frac{g}{s}\right)^{\alpha-2}\right]=1
$$

such that $g_{*}(s) \leq s$ for all $s \geq 0$,

$$
\lim _{s \rightarrow \infty} \frac{g_{*}(s)}{s}=1
$$

and $s_{*}$ is the root of the equation $g_{*}(s)=0$.

Remark. In case $\alpha=2$, equation (1.7) is considered to be of the form

$$
2 c g^{\prime} g \log \frac{s}{g}=1
$$

obtained from (1.7) by passing to the limit as $\alpha-2 \rightarrow 0$ (taking into account that $\left.\left[1-x^{\beta}\right] / \beta \rightarrow \log (1 / x), \beta \rightarrow 0, x>0\right)$.

Let us discuss a number of corollaries of Theorem 1 (see Figs. 1, 2 ).

Case $\alpha=1$. In this case, equation (1.7) becomes

$$
2 c g^{\prime}=\frac{1}{s-g} \text {. }
$$




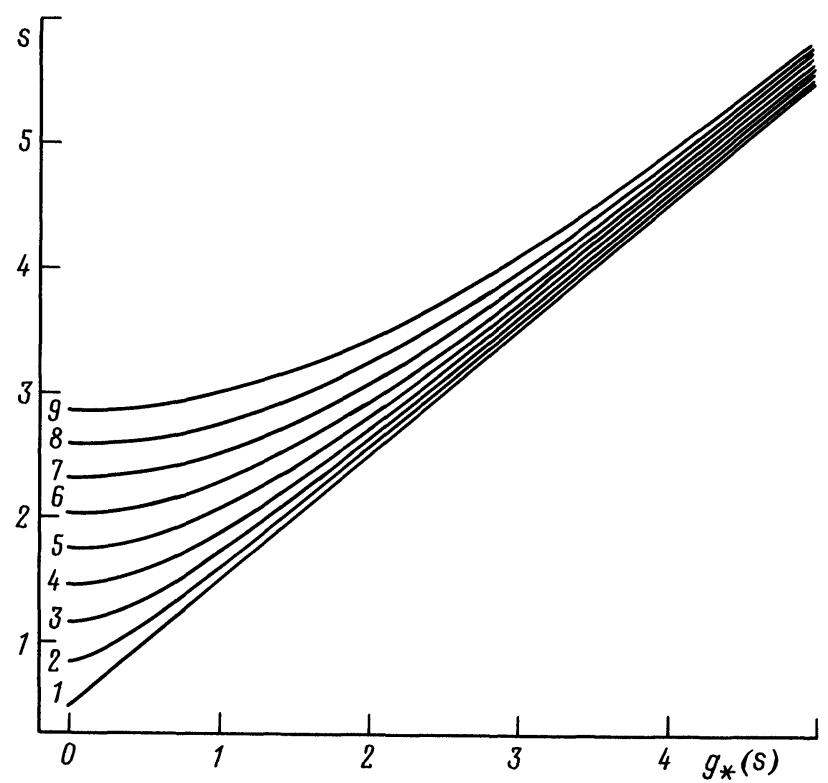

FIG. 1. Graphs of the curves $g_{*}=g_{*}(s)$ dividing the domains $D_{*}$ and $C_{*}$ for $c=1$ for the values of the parameter $\alpha=1,2, \ldots, 9$. (The computer computations for the solutions of equation (1.7) were carried out by John Overdeck,AT\&T Bell Laboratories.)

Let us rewrite this equation in the form

$$
\frac{d s}{d g}=\frac{1}{2 c}(s-g) .
$$

Its general solution has the form

$$
s(g)=\left(g+\frac{1}{2 c}\right)+K e^{g / 2 c},
$$

where $K$ are constants. It follows from this that the only solution which satisfies the condition $\lim _{g \rightarrow \infty}(s(g) / g)=1$ (compare with condition (1.8)) is the solution corresponding to the value $K=0$.

Thus in the given case $\alpha=1$, we obtain the result of [10]:

$$
g_{*}(s)=s-\frac{1}{2 c}
$$

"the critical" point is $s_{*}=1 /(2 c)$ and the optimal $\tau_{*}$ is

$$
\tau_{*}=\inf \left\{t: S_{t}-X_{t} \geq \frac{1}{2 c}\right\} .
$$

In other words, the observation should be stopped as soon as (a gap) $G_{t} \equiv S_{t}-X_{t}$ exceeds the value $1 /(2 c)$. It is clear that in the given case the "stopping domain" $D_{*}$ has the form

$$
D_{*}=\left\{(x, s): s \geq x+\frac{1}{2 c}\right\},
$$




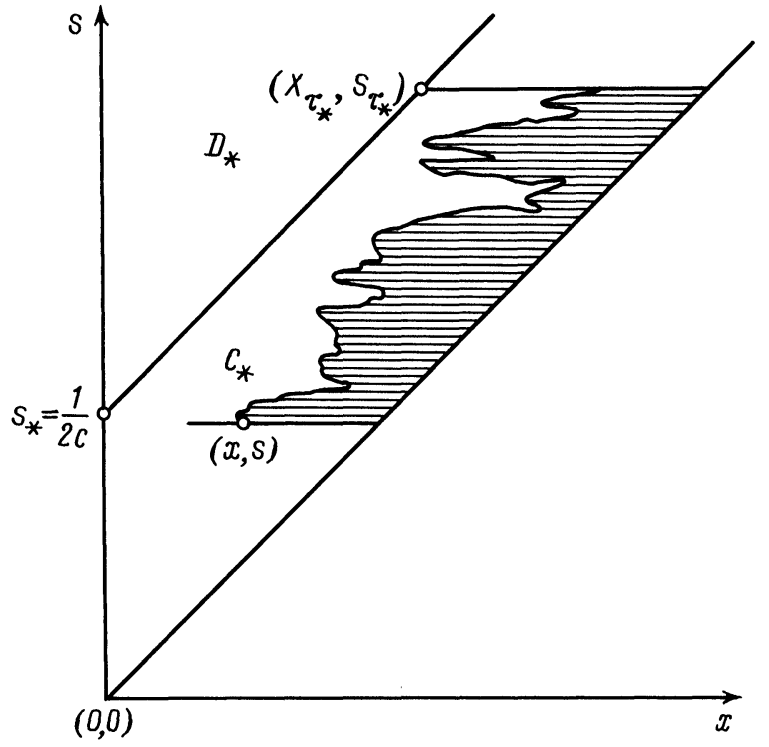

FIG. 2. The case $\alpha=1$. The stopping domain $D_{*}$ and the domain of continued observation $C_{*} ;$ the domain "covered" by the process $\left(X_{t}, S_{t}\right)$ for $0 \leq t \leq \tau_{*}$ and $\left(X_{0}, S_{0}\right)=(x, s)$ is hatched.

but the domain of continued observation $C_{*}=\{(x, s): s \geq x \geq 0\} \backslash D_{*}$ has (see Fig. 2) the following form here:

$$
C_{*}=\left\{(x, s): x \leq s<x+\frac{1}{2 c}\right\} .
$$

(We shall come back to a more detailed consideration of the case when $\alpha=1$ in $\S 3$.)

Case $\alpha \leq 0$. The point $x=0$ is a trap for the Bessel process $X \in \operatorname{Bes}^{\alpha}(0)$. Thus, if $X_{0}(0)=0$, then $X_{t}(0)=0$ for all $t \geq 0$ and the value $\tau_{*} \equiv 0$ is optimal for all $s \geq 0$. The corresponding equation, which determines the boundary between the domains $D_{*}$ and $C_{*}$, has in case $\alpha=0$ the form

$$
2 c g^{\prime}=\frac{1}{s-g}-\frac{1}{s+g}
$$

One can easily see that in this equation the chosen solution $g_{*}=g_{*}(s)$ (with the property $\left.\lim _{s \rightarrow \infty}\left(g_{*}(s) / s\right)=1\right)$ is such that $s-g_{*}(s) \rightarrow 1 /(2 c), s \rightarrow \infty$.

Case $0<\alpha<1$. In these cases, the point $x=0$ is a reflecting boundary for the Bessel processes $X \in \operatorname{Bes}^{\alpha}(x)$. Moreover, one can see from (1.7) that $g_{*}^{\prime}(0)=0$. Let us denote by $s_{*}(\alpha)$ the values of critical points $s_{*}$ (i.e., the roots of the equation $\left.g_{*}(s)=0\right)$ at the given values of $\alpha$. One can show that an increase of the values of $\alpha$ causes the increase of values $s_{*}(\alpha)$ as well. (The qualitative behavior of $g_{*}=g_{*}(s)$ boundaries at different values of $\alpha$ is given in Fig. 3.)

Case $\alpha>1$. If $1<\alpha<2$, then the point $x=0$ again (as in the case when $0<\alpha \leq 1$ ) is reflecting and is the entrance boundary for $\alpha \geq 2$. As is shown in (1.7), in these cases, $g_{*}^{\prime}(0)=\infty$. (See Fig. 3.)

The numerical calculation of $s_{*}(3)$ (at $c=1$ ) gives the following value:

$$
s_{*}(3) \approx 1.156 \ldots
$$

It is shown in $\S 6$ that $s_{*}(\alpha) \sim \alpha / 4$ as $\alpha \rightarrow \infty$ (Theorem 7$) ; s_{*}(\alpha)=0$ for $\alpha \leq 0$. 


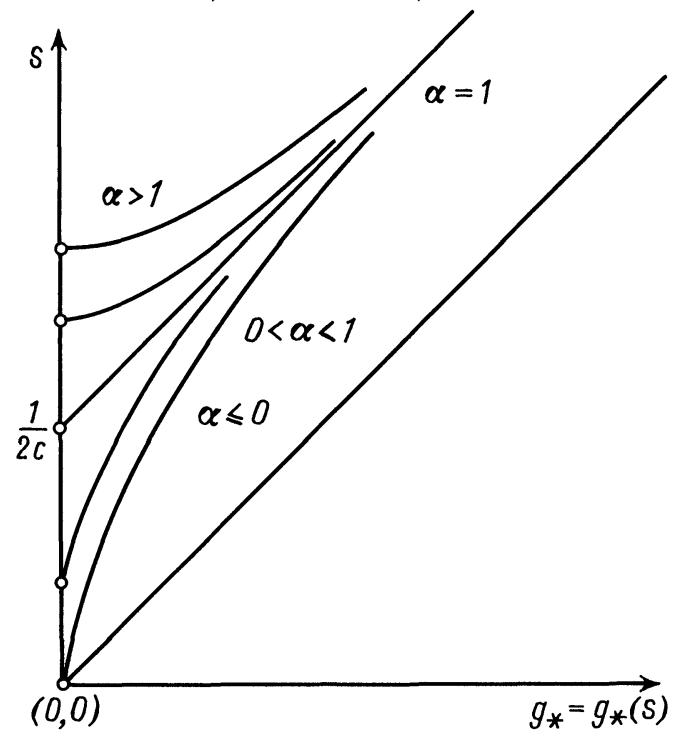

FIG. 3. A schematic drawing of the dividing curves (stopping boundaries) $g_{*}=g_{*}(s)$ for various values of the parameter $\alpha \in R$.

For greater clarity let us consider equations of the type (1.7) for a number of values of the parameter $\alpha$ :

$$
\begin{aligned}
2 c g^{\prime} & =\frac{3 g^{2}}{s^{3}-g^{3}}, & \alpha & =-1, \\
2 c g^{\prime} & =\frac{1}{s-g}-\frac{1}{s+g}, & \alpha & =0, \\
2 c g^{\prime} & =\frac{1}{s-g}, & \alpha & =1, \\
2 c g^{\prime} & =\frac{1}{g \log (s / g)}, & \alpha & =2, \\
2 c g^{\prime} & =\frac{1}{s-g}+\frac{1}{g}, & \alpha & =3 .
\end{aligned}
$$

3. In case $\alpha>0$, we represent the domain of continued observation $C_{*}$ as the sum of the two domains $C_{*}^{1}$ and $C_{*}^{2}$, where

$$
\begin{aligned}
& C_{*}^{1}=\left\{(x, s): g_{*}(s)<x \leq s \text { and } s_{*}<s\right\} \\
& C_{*}^{2}=\left\{(x, s): 0 \leq x \leq s \leq s_{*}\right\}
\end{aligned}
$$

(In case $\alpha \leq 0$, the value $s_{*}=0$, and the point $(0,0) \in D_{*}$ and $C_{*}^{1}=C_{*}$; the corresponding domain $C_{*}^{2}=\varnothing$.)

THEOREM 2. The value $V_{*}^{\alpha}=V_{*}^{\alpha}(x, s)$ is determined by the following formulas: if $\alpha>0$, then 
$V_{*}^{\alpha}(x, s)$

$$
= \begin{cases}s, & (x, s) \in D_{*}, \\ s+\frac{c}{\alpha}\left(x^{2}-g_{*}^{2}(s)\right)+\frac{2 c g_{*}^{2}(s)}{\alpha(\alpha-2)}\left[\left(\frac{g_{*}(s)}{x}\right)^{\alpha-2}-1\right], & (x, s) \in C_{*}^{1}, \\ \frac{c}{\alpha} x^{2}+s_{*}, & (x, s) \in C_{*}^{2}\end{cases}
$$

if $\alpha=0$, then

$$
V_{*}^{0}(x, s)= \begin{cases}s, & (x, s) \in D_{*}, \\ s+\frac{c}{2}\left[g_{*}^{2}(s)-x^{2}\right]+c x^{2} \log \frac{x}{g_{*}(s)}, & (x, s) \in C_{*}\end{cases}
$$

if $\alpha<0$, then

$V_{*}^{\alpha}(x, s)$

$$
= \begin{cases}s, & (x, s) \in D_{*}, \\ s+\frac{c}{\alpha}\left(x^{2}-g_{*}^{2}(s)\right)+\frac{2 c g_{*}^{2}(s)}{\alpha(\alpha-2)}\left[\left(\frac{g_{*}(s)}{x}\right)^{\alpha-2}-1\right], & (x, s) \in C_{*} .\end{cases}
$$

Remark. The formulas for $V_{*}^{0}(x, s)$ can be obtained through passage to the limit as $\alpha \downarrow 0$ in $V_{*}^{\alpha}(x, s)$, i.e., (1.14) follows from (1.13) as $\alpha \downarrow 0$. Similarly, in (1.13) the value $V_{*}^{\alpha}(x, s)$ for $\alpha=2$ must be understood as the result of passage to the limit as $\alpha \rightarrow 2$ which leads to the following result:

$$
V_{*}^{2}(x, s)= \begin{cases}s, & (x, s) \in D_{*}, \\ s+\frac{c}{2}\left(x^{2}-g_{*}^{2}(s)\right)+c g_{*}^{2}(s) \log \frac{g_{*}(s)}{x}, & (x, s) \in C_{*}^{1}, \\ \frac{c}{2} x^{2}+s_{*}, & (x, s) \in C_{*}^{2},\end{cases}
$$

where $s_{*}$ is the root of the equation $g_{*}(s)=0$, and $g_{*}=g_{*}(s)$ satisfies equation (1.9).

The proof of Theorems 1 and 2 in the case $\alpha>1$ is given in $\S 3 ; \S 4$ is devoted to the case when $\alpha=1$, where the (more general) problem of optimal stopping of Brownian motion with drift and (instantaneous) reflection is considered as well. The case when $\alpha<1$ is analyzed in $\S 5$. In $\S 6$ the results of optimal stopping are applied to the maximum inequalities for Bessel processes. It is shown that if $X \in \operatorname{Bes}^{\alpha}(0)$, then, for each stopping time $\tau$,

$$
\mathbf{E} \max _{u \leq \tau} X_{u} \leq \gamma(\alpha) \sqrt{\mathbf{E} \tau}
$$

where $\gamma(\alpha)=\sqrt{4 s_{1}(\alpha)}$, and $s_{1}(\alpha)$ is the root of the equation $g_{*}(s)=0$ for the function $g_{*}(s)$ which is the solution of equation (1.7) with $c=1$. It is also shown that $\gamma(\alpha) \sim \sqrt{\alpha}$ as $\alpha \rightarrow \infty$.

The authors of the paper express their gratitude to M. Yor for his productive consultations concerning Bessel processes.

The computer program has been carried out by N. Tolstoganova to whom the authors express their gratitude. 


\section{Bessel processes}

1. The systematic study of Bessel processes was initiated in McKean's paper [17]. The main sources of information concerning these processes are monographs [13], [14], [29], and, in particular, [20].

The simplest way of constructing a definition of Bessel processes $X \in \operatorname{Bes}^{\alpha}(x)$ is as follows.

Let $B=\left(B_{t}\right)_{t \geq 0}$ be Brownian motion on $\left(\Omega, \mathcal{F},\left(\mathcal{F}_{t}\right)_{t \geq 0}, \mathbf{P}\right)$. Let us consider the stochastic differential equation $(\alpha \in \mathbf{R})$

$$
d Y_{t}=\alpha d t+2 \sqrt{\left|Y_{t}\right|} d B_{t}, \quad Y_{0}=y \geq 0 .
$$

This equation has the only non-negative strong solution $Y_{t}=Y_{t}(y), t \geq 0$, i.e., such that, for each $t \geq 0$, the values of $Y_{t}$ are $\mathcal{F}_{t}^{B}$-measurable, where $\mathcal{F}_{t}^{B}=\sigma\left\{B_{s}, s \leq t\right\}$. According to [20], the process $Y=\left(Y_{t}(y)\right)_{t \geq 0}$ is called "the square of an $\alpha$-dimensional Bessel process" $\left(Y \in \operatorname{Besq}^{\alpha}(y)\right)$. The expression $\nu=\alpha / 2-1$ is called the index of the process. For $\alpha=0$, the process $Y$ is a martingale, for $\alpha<0$ it is a supermartingale, for $\alpha>0$ it is a submartingale.

The infinitesimal operator of the process is

$$
L=\alpha \frac{d}{d y}+2 y \frac{d^{2}}{d y^{2}} .
$$

The boundary point $y=0$ in case $\alpha \leq 0$ is a trap (or an absorbing point, [13, Chap. IV]), in the case where $0<\alpha<2$ it is the reflecting (instantaneously reflecting, [13, Chap. IV) boundary and it is the entrance boundary if $\alpha \geq 2$.

It should be noted that in the case where $\alpha=d=1,2, \ldots$ is an integer, the process $Y \in \operatorname{Besq}^{d}(y)$ permits the following obvious realization.

Let $B(a)=\left(B^{1}\left(a_{1}\right), \ldots, B^{d}\left(a_{d}\right)\right)$ be $d$-dimensional Brownian motion, starting at the point $a=\left(a_{1}, \ldots, a_{d}\right)$. Let us form the process $Y_{t}=Y_{t}\left(|a|^{2}\right), t \geq 0$, with

$$
Y_{t}\left(|a|^{2}\right)=\sum_{i=1}^{d}\left(B_{t}^{i}\left(a_{i}\right)\right)^{2}
$$

and assume that

$$
B_{t}=\sum_{i=1}^{d} \int_{0}^{t} \frac{B_{s}^{i}\left(a_{i}\right)}{Y_{s}\left(|a|^{2}\right)} d B_{s}^{i}\left(a_{i}\right)
$$

The process $B=\left(B_{t}\right)_{t \geq 0}$ is, obviously, one-dimensional standard Brownian motion. In this case, from Itô's formula it follows that $Y_{t}, t \geq 0$, satisfies the equation

$$
d Y_{t}=d \cdot d t+2 \sqrt{Y_{t}} d B_{t}
$$

Thus one can say that the square of the radial part of $d$-dimensional Brownian motion is "the square of a Bessel process of $d$-dimensionality".

2. Thus, let $Y \in \operatorname{Besq}^{\alpha}(y)$ for $y \geq 0$. We form the process

$$
X=\sqrt{Y} .
$$

In other words, let $X=\left(X_{t}(x)\right)_{t \geq 0}$ be a non-negative process such that $X_{t}(x)=$ $\sqrt{Y_{t}\left(x^{2}\right)}, x \geq 0$. 
The so-determined Markov process $X$ is the one that is called an $\alpha$-dimensional Bessel process $\left(X \in \operatorname{Bes}^{\alpha}(x)\right)$. Its generator is

$$
L=\frac{1}{2}\left(\frac{\alpha-1}{x} \frac{d}{d x}+\frac{d^{2}}{d x^{2}}\right),
$$

while the densities $p_{t}^{\alpha}(x, y)=\frac{d}{d y} \mathbf{P}\left(X_{t} \leq y \mid X_{0}=x\right)$ are expressed in terms of Bessel functions (see [20, p. 415]), which explains why the process is called the "Bessel" process.

Let us also note that for all $\alpha \geq 1$ the Bessel processes are (non-negative) submartingales.

In case $\alpha \geq 2$, the boundary point $x=0$ is the entrance boundary, which allows one to use the Itô formula for $\sqrt{Y_{t}\left(x^{2}\right)}$ which for $X_{t}(x)=\sqrt{Y_{t}\left(x^{2}\right)}$ leads to the following stochastic differential equation:

$$
d X_{t}(x)=\frac{\alpha-1}{2} \frac{d t}{X_{t}(x)}+d B_{t}, \quad X_{0}(x)=x \geq 0
$$

This equation has a strong solution not only for $\alpha \geq 2$ but also for all $\alpha>1$ (see [20]), which coincides with $\sqrt{Y}, Y \in \operatorname{Besq}^{\alpha}\left(x^{2}\right)$. Thus equation (2.6) can be considered to be a constructive method of describing Bessel processes for $\alpha>1$. The situation is more complicated for $\alpha \leq 1$. If $\alpha=1$, then the corresponding Markov process $X(x)$ has the infinitesimal operator $L=\frac{1}{2} d^{2} / d x^{2}$, and the point $x=0$ is the reflecting boundary. It is well known (see [20]), that such a process can be realized in the form $X_{t}(x)=\left|B_{t}+x\right|$, i.e., as the Brownian motion, starting at the point $x \geq 0$ with reflection at $x=\{0\}$. The corresponding analogue of equation (2.6) here is the stochastic differential equation with reflection:

$$
d X_{t}=d L_{t}^{0}(X)+d B_{t}, \quad X_{0}(x)=x,
$$

where $L_{t}^{0}(X)$ is the local time of the process $X$ at zero; see also $\S 4$. If $0<\alpha<1$, then in this case (unlike the case $\alpha \geq 1$ ) the Bessel processes $X(x) \in \operatorname{Bes}^{\alpha}(x)$ are not semimartingales (i.e., processes which do not permit a representation as a sum of a local martingale and a process of locally bounded variation). However, they still permit a representation of the form

$$
X_{t}(x)=x+B_{t}+\frac{(\alpha-1)}{2} H_{t}
$$

where again $B=\left(B_{t}\right)_{t \geq 0}$ is a standard Brownian motion, and $H=\left(H_{t}\right)_{t \geq 0}$ is a process of locally zero quadratic variation. (In [11], [12] such processes are called Dirichlet processes.) Moreover, the process $H$ can be described as follows ([2]-[5], $[26])$ :

$$
H_{t}=\mathrm{p} . \mathrm{v} \cdot \int_{0}^{t} \frac{d s}{X_{s}(x)} \stackrel{\mathrm{d}}{=} \lim _{\varepsilon \downarrow 0} \int_{0}^{t} \frac{I\left(X_{s}>\varepsilon\right)}{X_{s}(x)} d s
$$

Let $\left\{L_{t}^{a}, a \geq 0\right\}$ be a family of local times of the process $X$. It is well known (see [20]) that for each non-negative Borel function $\varphi=\varphi(a)$, for $a \geq 0$,

$$
\int_{0}^{t} \varphi\left(X_{s}\right) d s=\int_{0}^{\infty} \varphi(a) a^{\alpha-1} L_{t}^{\alpha}(d a) .
$$


Taking this property into account, the process $H=\left(H_{t}\right)_{t \geq 0}$ (as "a principal value" in the sense of Hadamard [2, p. 232] and [26, p. 121]), can be defined in the following way:

$$
H_{t}=\lim _{\varepsilon \downarrow 0} \int_{\varepsilon}^{\infty} L_{t}^{0} a^{\alpha-2} d a=\int_{0}^{\infty}\left(L_{t}^{a}-L_{t}^{0}\right) a^{\alpha-2} d a .
$$

In case $\alpha=0$, the point $x=0$ is a trap (absorbing point) for the process $X \in \operatorname{Bes}^{0}(x), x>0$. In this case the square of the process, $Y=X^{2}$, satisfies the equation

$$
d Y_{t}=2 \sqrt{Y_{t}} d B_{t}
$$

and is a martingale.

In case $\alpha<0$, the corresponding equation for $Y=X^{2}$ has the form

$$
d Y_{t}=\alpha d t+2 \sqrt{Y_{t}} d B_{t}
$$

and, consequently, this process is a non-negative submartingale which after reaching the point $x=0$ stays there. (These remarks explain M. Yor's point of view that in case $\alpha \leq 0$ the study of the properties of the process $X$ can be easily carried out by studying the properties of its square, $Y=X^{2}$.)

\section{The case when $\alpha>1$}

1. Let $X \in \operatorname{Bes}^{\alpha}(x)$, where $\alpha \geq 0, x \geq 0, X_{t}=X_{t}(x), S_{t}=S_{t}(x, s), x \leq s$. We denote

$$
V(x, s)=\sup _{\tau} \mathbf{E}_{x, s}\left[S_{\tau}-c \tau\right]
$$

where the sup is taken over all finite stopping times $\tau$, and $\mathbf{E}_{x, s}$ means the expectation under the assumption $X_{0}(x)=x, S_{0}(x, s)=s, x \leq s$. (We now reserve the notation $V_{*}^{\alpha}(x, s)$ for the function given in Theorem 2.)

From (3.1) it follows that $V(x, s) \geq s$. If $\alpha=d$ is an integer, then from (2.3) we find that, for each finite stopping time $\tau$,

$$
\mathbf{E}_{x, s} S_{\tau}=\mathbf{E} S_{\tau}(x, s) \leq s \vee \mathbf{E} \max _{r \leq \tau} X_{r}(x) \leq s \vee d\left(\mathbf{E} \sup _{r \leq \tau}\left|B_{r}^{1}\right|+x\right) .
$$

According to Doob's inequality, $\mathbf{E s u p}_{r \leq \tau}\left|B_{r}^{1}\right| \leq 2 \sqrt{\mathbf{E} \tau}$. Therefore, from (3.1) it follows that

$$
\mathbf{E}_{x, s}\left[S_{\tau}-c \tau\right] \leq s+d x+2 d \sqrt{\mathbf{E} \tau}-c \mathbf{E} \tau
$$

It is clear from this, that in taking the sup of $\tau$ in (3.1) it is sufficient (in the case of integral $\alpha=d=1,2, \ldots)$ to consider only $\tau$ with $\mathbf{E} \tau<\infty$.

From (3.2) it also follows that

$$
\sup _{\tau} \mathbf{E}\left[S_{\tau}(x, s)-c \tau\right] \leq s+d \cdot x+\frac{d^{2}}{c} .
$$

Thus (for integral $\alpha=d=1,2, \ldots$ )

$$
s \leq V(x, s) \leq s+d \cdot x+\frac{d^{2}}{c} \leq s[1+d]+\frac{d^{2}}{c} .
$$


Using the comparison theorem (see [20, Chap. IX]) for solutions $Y \in \operatorname{Besq}^{\alpha}(y)$ and $Y \in \operatorname{Besq}^{[\alpha]+1}(y)$ of equations (2.1), we find that for each $\alpha$ sup in (3.1), it is sufficient to use only those $\tau$ for which $\mathbf{E} \tau<\infty$ and (3.3) holds with $d=[\alpha \wedge 0]+1$.

2. From (3.1) we find that (with the function $V_{*}^{\alpha}(x, s)$, given in Theorem 2)

$$
\begin{aligned}
V(x, s) & \leq \sup _{\tau} \mathbf{E}_{x, s}\left[S_{\tau}-V_{*}^{\alpha}\left(X_{\tau}, S_{\tau}\right)\right]+\sup _{\tau} \mathbf{E}_{x, s}\left[V_{*}^{\alpha}\left(X_{\tau}, S_{\tau}\right)-c \tau\right] \\
& \leq \sup _{\tau} \mathbf{E}_{x, s}\left[V_{*}^{\alpha}\left(X_{\tau}, S_{\tau}\right)-c \tau\right]
\end{aligned}
$$

where we used the fact that $V_{*}^{\alpha}(x, s) \geq s$. Therefore, to prove Theorems 1 and 2 it is sufficient to show that

$\left(A_{1}\right)$ for each (admissible) stopping time $\tau$ (with $\left.\mathbf{E} \tau<\infty\right)$,

$$
\mathbf{E}_{x, s}\left[V_{*}^{\alpha}\left(X_{\tau}, S_{\tau}\right)-c \tau\right] \leq V_{*}^{\alpha}(x, s), \quad 0 \leq x \leq s
$$

and

$\left(A_{2}\right)$ for the stopping time $\tau_{*}$, given in Theorem 1 ,

$$
\mathbf{E}_{x, s}\left[S_{\tau_{*}}-c \tau_{*}\right]=V_{*}^{\alpha}(x, s), \quad 0 \leq x \leq s .
$$

Before checking the validity of properties $\left(A_{1}\right)$ and $\left(A_{2}\right)$ let us make a number of preliminary considerations, which explain the appearance of equations (1.7) and the form of the functions $V_{*}^{\alpha}(x, s)$ in the conditions of Theorem 2 .

Finding "the value" $V(x, s)$ in problem (3.1) can, generally speaking, be carried out within the common theory of optimal stopping rules of the Markov processes with continuous time (see, for example, [28], [27], [33]). Without specifying the exact corresponding formulations concerning the possibilities of applying the general theory to the Markov process $(X, S)$ under consideration (eventually when establishing the $\left(A_{1}\right)$ and $\left(A_{2}\right)$ properties we shall act otherwise, using the results of the general "Markov" theory of optimal stopping as guidelines in combination with "martingale" methods), let us remember, that the value $V(x, s)$ satisfies, for each point $(x, s)$, the equation

$$
V(x, s)=\max \left\{V_{0}(x, s),\left(T_{\tau(v)} V\right)(x, s)-c \mathbf{E}_{x, s} \tau(v)\right\},
$$

where $v=v(x, s)$ is "the sufficient by small" neighborhood of the point $(x, s), \tau(v)$ is the time of the first exit of the process $\left(X_{t}(x), S_{t}(x, s)\right)_{t \geq 0}$ from this neighborhood, $\left(T_{\tau(v)} V\right)(x, s)=\mathbf{E}_{x, s} V\left(X_{\tau(v)}(x), S_{\tau(v)}(x, s)\right)$ and (in the given case) $V_{0}(x, s)=s$ is the gain from stopping without observation. (See, for example, [27, Thm. 5] and [33, Chap. III].)

It is also known ([28], [27], [33]), that in "regular" cases the optimal stopping time $\tau_{*}$ has the following structure:

$$
\tau_{*}=\inf \left\{t: V\left(X_{t}, S_{t}\right)=V_{0}\left(X_{t}, S_{t}\right)\right\}
$$

i.e., $\tau_{*}$ is the time of the first hit in the "stopping domain"

$$
D_{*}=\left\{(x, s): V(x, s)=V_{0}(x, s)\right\} .
$$

In the domain of "continued observation"

$$
C_{*}=\left\{(x, s): V(x, s)>V_{0}(x, s)\right\},
$$


it follows from (3.7) that the value $V(x, s)$ satisfies the equation

$$
V(x, s)=\left(T_{\tau(v)} V\right)(x, s)-c \mathbf{E}_{x, s} \tau(v)
$$

which implies that

$$
\mathrm{A} V(x, s)=c,
$$

where A is a characteristic operator of the Markov process $(X, S)$ (see [29] and [33, Chap. III, §8].)

In connection with (3.9) let us consider the structure of the process $(X, S)=$ $\left(X_{t}(x), S_{t}(x, s)\right)_{t \geq 0}$ in greater detail.

If, for $t=0, X_{0}(x)=x, S_{0}(x, s)=s$, where $x<s$, then the process $(X, S)$ begins moving along the horizontal $H_{s}=\{(y, s): 0 \leq y \leq s\}$. So that until the time $\sigma_{s}=\inf \left\{t: X_{t}=s\right\}$ only the first component $X$ of the process $(X, S)$ is changed, and the second component being unchanged remains equal to $s$. Getting to the diagonal $\Delta=\{(x, s): x=s\}$, the process $(X, S)$ begins to move in a strange way both along the diagonal upwards and leaving it and starting to move along higher horizontal lines.

Thus, in a certain sense, the two-dimensional Markov process $(X, S)$ considered is degenerate (wandering along horizontal lines for $x<s$ with a "rise" to higher horizontal lines at the times of getting into the diagonal $\Delta=\{(x, s): x=s\}$ ).

Let us also notice that the component $X$ is a diffusion type process. At the same time, the component $S$ is the process of a locally bounded (almost surely) variation. It is interesting to emphasize that the degeneration of the process $(X, S)$ considered turns out to be, as it will become clear from further analysis, a favourable circumstance which allows one to give a closed solution of the optimal stopping problem in the "twodimensional problem (3.1)".

3. In the domain of continuation of observations $C_{*}$ the value $V(x, s)$ satisfies equation (3.9). Therefore, if $x<s$ and it is a priori known that the function $V(x, s)$ is sufficiently smooth on $x$, then equation (3.9) becomes the equation

$$
L V(x, s)=c,
$$

where $L$ is the infinitesimal operator of the process $X$, defined by formula (1.1), i.e.,

$$
\frac{\alpha-1}{2 x} \frac{\partial V(x, s)}{\partial x}+\frac{1}{2} \frac{\partial^{2} V(x, s)}{\partial x^{2}}=c, \quad 0<x<s .
$$

The general solution of this equation (for a fixed $s$ ) is defined, as one can easily see, by the following formulas:

if $\alpha=0$, then

$$
V(x, s)=c x^{2} \log x+a(s) x^{2}+b(s)
$$

if $\alpha=2$, then

$$
V(x, s)=\frac{c}{2} x^{2}+a(s) \log x+b(s)
$$

if $\alpha(\alpha-2) \neq 0$, then

$$
V(x, s)=\frac{c}{\alpha} x^{2}+\frac{a(s)}{x^{\alpha-2}}+b(s),
$$

where $a(s)$ and $b(s)$ are undefined (for each $s$ ) constants, subject to a definition. 


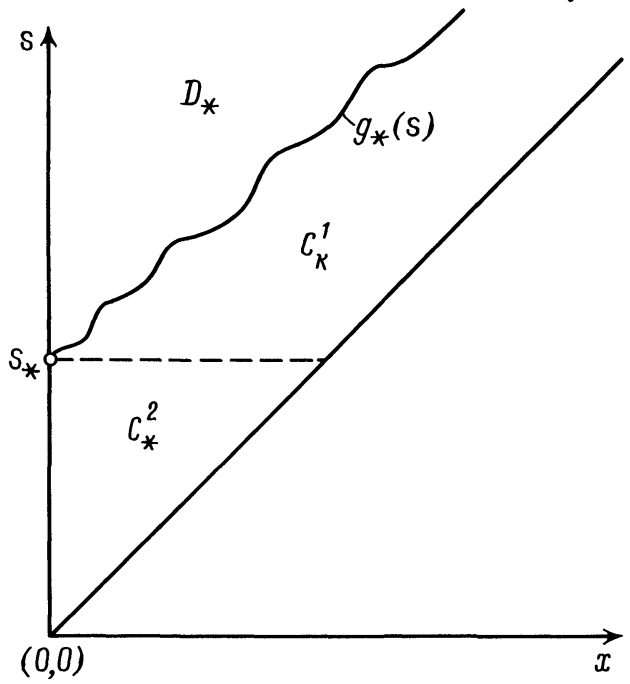

FIG. 4. The dividing curve $g_{*}(s)$, the stopping domain $D_{*}$ and the domain of continued observation $C_{*}=C_{*}^{1} \cup C_{*}^{2}$.

Considerations leading to their determination, consist of the following.

Equation (3.10), determining $V(x, s)$, "acts" in the domain of "continued observation" $C_{*}$, which, as follows from the general theory, must have the form

$$
C_{*}=\{(x, s): V(x, s)>s\}
$$

and thus being dependent on an unknown function $V(x, s)$, is unknown as well.

The guideline for the next step is the leading idea that the domains $C_{*}$ and $D_{*}=\{(x, s): V(x, s)=s\}$ are divided by some (for the present unknown) boundary $g_{*}=g_{*}(s)$, where $D_{*}$ has the following form (see Fig. 4):

$$
D_{*}=\left\{(x, s): 0 \leq x \leq s, s_{*} \leq s, x \leq g_{*}(s)\right\} \text {. }
$$

An intuitive argument for the likelihood of existence of such a dividing boundary consists of the following.

If some point $\left(x_{0}, s_{0}\right)$ belongs to $D_{*}$, then all the points $(x, s)$ with $x \leq x_{0}$ and $s \geq s_{0}$ must (according to the meaning of problem (1.4) considered) also belong to $D_{*}$. (Recall that in case $\alpha \leq 0$ the point $x_{0}=0$ is a trap for $X \in \operatorname{Bes}^{\alpha}(0)$ and, consequently, all the points $\left(0, s_{0}\right), s_{0} \geq 0$, belong to the domain $D_{*}$.) Further, if one supposes that the point of the diagonal $\left(x_{0}, s_{0}\right)$ with $x_{0}=s_{0}>0$ belongs to the domain $D_{*}$, then, consequently, the observation must be stopped immediately and the corresponding value $V\left(s_{0}, s_{0}\right)=s_{0}$. Let us consider, however, the stopping rule according to which an observation takes place during some small interval of time $\Delta$ and at the time $\Delta$ the observation is stopped. Then up to a stochastic quantity of order $o(\Delta)$ the quantity $S_{\Delta}=s_{0} \vee \max _{u \leq \Delta} X_{u}$ coincides with $s_{0} \vee\left(s_{0}+\Delta X\right)$, where $\Delta X=X_{\Delta}-X_{0}$. If $\Delta X \leq 0$, then $S_{\Delta}=s_{0}+o(\Delta)$; but if $\Delta X>0$, then $S_{\Delta}=s_{0}+\Delta X+o(\Delta)$. Hence, one can conclude that

$$
\begin{aligned}
\mathbf{E}_{s_{0}, s_{0}}\left[S_{\Delta}-c \Delta\right] & =s_{0}+\mathbf{E}_{s_{0}, s_{0}}\left[S_{\Delta} ; \Delta X \leq 0\right]+\mathbf{E}_{s_{0}, s_{0}}\left[S_{\Delta} ; \Delta X>0\right]-c \Delta+o(\Delta) \\
& =s_{0}+\mathbf{E}_{s_{0}, s_{0}}[\Delta X ; \Delta X>0]-c \Delta+o(\Delta) \\
& =s_{0}+\sqrt{\frac{\Delta}{2 \pi}}-c \Delta+o(\Delta),
\end{aligned}
$$


where we used the fact that, for $X \in \operatorname{Bes}^{\alpha}\left(s_{0}\right), s_{0}>0$,

$$
\mathbf{E}_{s_{0}}(\Delta X ; \Delta X>0) \equiv \mathbf{E}_{s_{0}} \Delta X I(\Delta X>0) \sim \sqrt{\frac{\Delta}{2 \pi}} .
$$

In view of the fact that the right part of (3.14) contains the quantity $\sqrt{\Delta}$, we see that at least for rather small $\Delta$ it is more advantageous not to stop the observation instantaneously (at the point $\left(s_{0}, s_{0}\right), s_{0}>0$ ) but to realize the observations. Thus the points of the diagonal $\left(s_{0}, s_{0}\right), s_{0}>0$, must belong to the domain of continuation of the observation. (If $\alpha>0$, then the point $(0,0)$ also belongs to $C_{*}$.)

Along with the unknown functions $a(s), b(s)$, in (3.11)-(3.13), the boundary $g_{*}=g_{*}(s)$ is also unknown and, consequently, we need at least three conditions for their determination. One of these conditions that of the continuity of the value at the boundary is "quite obvious":

$$
\left.V(x, s)\right|_{x \uparrow g_{*}(s)}=s .
$$

The condition of "smooth pasting" used later,

$$
\left.\frac{\partial V(x, s)}{\partial x}\right|_{x \uparrow g_{*}(s)}=0,
$$

is less obvious, it states that the derivative of the value with respect to $x$ on approaching the boundary must coincide with the corresponding derivative of the gain, obtained from the instantaneous stopping (i.e., coincide with $\left.\frac{\partial V_{0}(x, s)}{\partial x}\right|_{x \downarrow g_{*}(s)}$, which in the given case is equal to zero, since $\left.V_{0}(x, s)=s\right)$.

Remark. As far as we know the condition of "smooth pasting" was used for the solution of concrete problems of statistical sequential analysis in the thesis of V. S. Mikhalevich (1955) (see also [19]) and A. N. Shiryaev (1961), done in Moscow under the leadership of A. N. Kolmogorov. This condition was used in 1961 in the works of H. Chernoff [6] and D. Lindley [16], and in 1965, in the works of P. Samuelson [22] and H. McKean [18] in connection with options of "American type". In the work of V. Benesh, L. Shepp and G. Witsenhauser [1] applications and (and a survey) of this condition to problems of optimal control are given. See also $\S 38$ in [33], where one can find a number of references to corresponding publications, and the paper [27], devoted to a systematic study of analytical methods of the solution of problems of optimal stopping by means of their reduction to "Stephan's problems" (for parabolic equations with moving boundaries).

Finally, the third condition of normal "reflection" on a diagonal used later

$$
\left.\frac{\partial V(x, s)}{\partial s}\right|_{s=x}=0
$$

is suggested by the following considerations based as in (3.15) on "the effect of $\sqrt{\Delta}$ ".

Let us suppose that the value $V(x, s)$ is a rather smooth function. Then considering the evolution of the function $V\left(X_{t}, S_{t}\right)$ for $t \leq \Delta$ and $X_{0}=s, S_{0}=s, s>0$, we come to the following expression based on the formula of complete probability and Tailor's decomposition:

$$
\begin{aligned}
V(s, s)= & -c \Delta+\mathbf{E}_{s, s}[V(s+\Delta X, s) \mid \Delta X \leq 0] P_{s, s}(\Delta X \leq 0) \\
& +\mathbf{E}_{s, s}[V(s+\Delta X, s+\Delta X) \mid \Delta X>0] P_{s, s}(\Delta X>0)+0(\Delta)
\end{aligned}
$$




$$
\begin{aligned}
= & -c \Delta+V(s, s)+\frac{\partial V}{\partial x}(s, s) \mathbf{E}_{s, s} \Delta X+\frac{1}{2} \frac{\partial^{2} V}{\partial x^{2}} \mathbf{E}_{s, s}(\Delta X)^{2} \\
& +\frac{\partial V}{\partial s}(s, s) \mathbf{E}(\Delta X ; \Delta X>0)+o(\Delta) .
\end{aligned}
$$

Since $\mathbf{E}_{s, s} \Delta X \sim \frac{\alpha-1}{2 s} \Delta, \mathbf{E}_{s, s}(\Delta X)^{2} \sim \Delta$ and $\mathbf{E}_{s, s}(\Delta X ; \Delta X>0) \sim \sqrt{\Delta /(2 \pi)}$, we find from (3.19) that

$$
-c \sqrt{\Delta}+\frac{\partial V}{\partial x}(s, s) \frac{\alpha-1}{2 s} \sqrt{\Delta}+\frac{1}{2} \frac{\partial^{2} V}{\partial x^{2}} \sqrt{\Delta}+\frac{\partial V}{\partial s}(s, s) \sqrt{\frac{1}{2 \pi}}+o(\sqrt{\Delta})=0 .
$$

It is clear from this that passage to the limit as $\Delta \rightarrow 0$ leads to the condition (3.18). (This condition can also be obtained by the methods used in the proof of Theorem 8 in [27].)

Thus for the determination of the three unknown functions $a(s), b(s)$, and $g_{*}(s)$ we have three conditions (3.16), (3.17), (3.18).

At first let $\alpha(\alpha-2) \neq 0$. Then from (3.13) and (3.16), we have

$$
\frac{c}{\alpha} g_{*}^{2}(s)+\frac{a(s)}{g_{*}^{\alpha-2}(s)}+b(s)=s .
$$

Condition (3.17) gives

$$
\frac{2 c}{\alpha} g_{*}(s)+\frac{(2-\alpha) a(s)}{g_{*}^{\alpha-1}(s)}=0 .
$$

Therefore,

$$
a(s)=\frac{2 c g_{*}^{\alpha}(s)}{\alpha(\alpha-2)}
$$

and

$$
b(s)=s-\frac{c}{\alpha-2} g_{*}(s) .
$$

Thus for $g_{*}(s)<x \leq s$ (i.e., in the domain $C_{*}^{1}$ in accordance with Fig. 4) for $\alpha(\alpha-2) \neq 0$,

$$
V_{*}^{\alpha}(x, s)=s+\frac{c}{\alpha}\left(x^{2}-g_{*}^{2}(s)\right)+\frac{2 c g_{*}^{2}(s)}{\alpha(\alpha-2)}\left[\left(\frac{g_{*}(s)}{x}\right)^{\alpha-2}-1\right] .
$$

Finally, taking into account condition (3.18), we find that $g_{*}=g_{*}(s)$ is a solution of the equation

$$
\frac{2 c}{\alpha-2} g(s) g^{\prime}(s)\left[1-\left(\frac{g(s)}{s}\right)^{\alpha-2}\right]=1 .
$$

Similar considerations for the case $\alpha=0$ lead to the same equation (3.23). In case $\alpha=2$ we find from (3.12) and (3.16)-(3.18) that $g_{*}=g_{*}(s)$ is the solution of the equation

$$
2 c g(s) g^{\prime}(s) \log \frac{s}{g(s)}=1
$$

which, as has been already mentioned, belongs to the family (3.23), understood, for $\alpha=2$, as the result of formal passage to the limit as $\alpha-2 \rightarrow 0$ (taking into account that $\left.\left[1-x^{\beta}\right] / \beta \rightarrow \log (1 / x), \beta \rightarrow 0, x>0\right)$. 
In case $\alpha(\alpha-2)=0$, the corresponding expressions for $V_{*}^{\alpha}(x, s)$ in the domain $C_{*}^{1}$ are obtained analogously and are given in (1.14), (1.16).

If $\alpha=1$, then equation (3.23) takes the form

$$
2 c g^{\prime}(s)=\frac{1}{s-g(s)}
$$

the general solution of which was described in $\S 1$, Corollary 1 to Theorem 1 . In the general case $(\alpha \neq 1)$, it is probably impossible to find solutions of equations (3.23) in closed form. However, we can give additional conditions, which allow us to "reduce" the set of solutions, among which we have to look for "the needed" solution $g_{*}=g_{*}(s)$.

In fact, it has already been mentioned in $\S 1$ that the value $V_{*}^{\alpha}(x, s)$ satisfies the inequalities

$$
s \leq V_{*}^{\alpha}(s, s) \leq s[1+d]+\frac{d^{2}}{c}
$$

with $d=[\alpha \vee 0]+1$.

If $\alpha(\alpha-2) \neq 0$, then from (3.22) and (3.26) we find, denoting $h_{*}(s)=g_{*}(s) / s$, that if $V_{*}^{\alpha}(x, s)$ is determined by means of formula (3.22), then the inequalities

$$
\frac{1}{s} \leq \frac{1}{s}+\frac{c}{\alpha}\left[1-h_{*}^{2}(s)\right]+\frac{2 c h_{*}^{2}(s)}{\alpha(\alpha-2)}\left[h_{*}^{\alpha-2}(s)-1\right] \leq \frac{1+d}{s}+\frac{d^{2}}{2 c s^{2}}
$$

must hold. Assume now for simplicity of considerations that $c=1$ and $\alpha=d=3$ (the considerations in the general case are analogous). Then (3.27) becomes the inequality

$$
\frac{1}{s} \leq \frac{1}{s}+\frac{1}{3}+\frac{2}{3} h_{*}^{3}(s)-h_{*}^{2}(s) \leq \frac{4}{s}+\frac{9}{s^{2}}
$$

from which it follows that

$$
\frac{1}{3}+\limsup _{s \rightarrow \infty}\left[\left(\frac{2}{3}\right) h_{*}^{3}(s)-h_{*}^{2}(s)\right]=0
$$

and

$$
\frac{1}{3}+\liminf _{s \rightarrow \infty}\left[\left(\frac{2}{3}\right) h_{*}^{3}(s)-h_{*}^{2}(s)\right]=0 .
$$

Let $\bar{h}=\lim \sup _{s \rightarrow \infty} h_{*}(s)$ and $\underline{h}=\lim _{\inf _{s \rightarrow \infty}} h_{*}(s)$. Then from the two last relations we find that

$$
\frac{1}{3}+\frac{2}{3} \bar{h}^{3}-\underline{h}^{2}=0, \quad \frac{1}{3}+\frac{2}{3} \underline{h}^{3}-\bar{h}^{2}=0
$$

and, consequently,

$$
\frac{2}{3} \bar{h}^{3}+\bar{h}^{2}=\frac{2}{3} \underline{h}^{3}+\underline{h}^{2} .
$$

Since one must have $g_{*}(s) \leq s$, then $\underline{h} \leq \bar{h} \leq 1$ and it follows from (3.29) that $\bar{h}=\underline{h}$. Denoting $a=\bar{h}$ we find from $(3.28)$ that $(a-1)^{2}\left(a+\frac{1}{2}\right)=0$. Consequently, $\underline{h}=\bar{h}=1$, i.e., "the optimal" boundary $g_{*}=g_{*}(s)$, dividing the domains of stopping the observation $D_{*}$ and of continuing the observation $C_{*}$, must satisfy the property

$$
\lim _{s \rightarrow \infty} \frac{g_{*}(s)}{s}=1
$$


which gives the condition at infinity for equations (3.23) and (3.24) and selects in the family of their solutions that solution (moreover, the only one) which is in keeping with the problem under consideration. (The existence and uniqueness of such a solution can be obtained by standard methods of the theory of differential equations of first order, see, for example, [30, Part 1, Chap. I].)

4. Let $s_{*}=s_{*}(\alpha)$ be the root of the equation $g_{*}(s)=0$, where $g_{*}=g_{*}(s)$ is the solution of equation (1.7), satisfying condition (3.30) at infinity.

In accordance with Fig. 4 and the above arguments, in the domain $C_{*}^{1}$ the function $V_{*}^{\alpha}(x, s)$ is given by formula (3.22). If the observation begins at the point $(x, s) \in C_{*}^{2}$, then (with the exception of the point $(0,0)$ which is a trap for $\alpha \leq 0$ ) stopping of the observation can be realized only after getting into the domain $C_{*}^{1}$ by passing through the point $\left(s_{*}, s_{*}\right)$.

Therefore, if $V_{*}^{\alpha}\left(s_{*}, s_{*}\right)$ is the value under the assumption that the observation begins at the point $\left(s_{*}, s_{*}\right)$, then the value $V_{*}^{\alpha}(x, s)$ for $(x, s) \in C_{*}^{2}$ and $\alpha>0$ will be determined by

$$
V_{*}^{\alpha}(x, s)=V_{*}^{\alpha}\left(s_{*}, s_{*}\right)-c \mathbf{E}_{x, s} \sigma_{*},
$$

where $\sigma_{*}=\inf \left\{t:\left(X_{t}, S_{t}\right)=\left(s_{*}, s_{*}\right)\right\}$.

The quantity $\mathbf{E}_{x, s} \sigma_{*}$ can be determined from the following considerations.

Let $X \in \operatorname{Bes}^{\alpha}(x), \alpha>0$. Then the process $Y=X^{2}$ satisfies equation (2.1) and, consequently, the process $\left(Y_{t}-Y_{0}-\alpha t\right)_{t \geq 0}$ is a martingale.

Thus, for each $t>0$,

$$
\mathbf{E}_{x, s}\left[Y_{\sigma_{*} \wedge t}-x^{2}-\alpha\left(\sigma_{*} \wedge t\right)\right]=0 .
$$

Then

$$
\alpha \mathbf{E}_{x, s}\left(\sigma_{*} \wedge t\right)=\mathbf{E}_{x, s}\left[Y_{\sigma_{*} \wedge t}-x^{2}\right] \quad\left(\leq s_{*}^{2}-x^{2}\right)
$$

and, according to the Fatou lemma,

$$
\mathbf{E}_{x, s} \sigma_{*} \leq \frac{s_{*}^{2}-x^{2}}{\alpha}<\infty
$$

The finiteness of $\mathbf{E}_{x, s} \sigma_{*}$ enables one to go to the limit as $t \rightarrow \infty$ in (3.32) which gives for $\mathbf{E}_{x, s} \sigma_{*}$ the following expression:

$$
\mathbf{E}_{x, s} \sigma_{*}=\frac{s_{*}^{2}-x^{2}}{\alpha}
$$

Since $g_{*}\left(s_{*}\right)=0$, we have from $(3.22)$

$$
V_{*}^{\alpha}\left(s_{*}, s_{*}\right)=s_{*}+\frac{c}{\alpha} s_{*}^{2} .
$$

Together with (3.31) this yields (for each point $(x, s) \in C_{*}^{2}$ and $\alpha>0$ )

$$
V_{*}^{\alpha}(x, s)=s_{*}+\frac{c}{\alpha} x^{2},
$$

which explains the appearance of this expression in (1.13) in case $(x, s) \in C_{*}^{2}$.

5. After these preliminary remarks let us proceed directly to the proofs of Theorems 1 and 2 for the case when $\alpha>1$. 
In this case the Bessel processes $X \in \operatorname{Bes}^{\alpha}(x)$ may be considered as solutions of the stochastic differential equation (2.6).

The process $(X, S)$ is a two-dimensional semimartingale, where the first component is a diffusion-type process, and the second a process of locally bounded variation. For the function $V_{*}^{\alpha}\left(X_{t}, S_{t}\right)$, where $V_{*}^{\alpha}(x, s)$ is given in (1.13), it is possible to apply the Itô formula ([20, Chap. IV,$\S 3] ;[13]$ ), which gives the expression (omitting the index $\alpha$ in $V_{*}^{\alpha}$ for simplicity)

$$
\begin{aligned}
V_{*}\left(X_{t}, S_{t}\right)= & V_{*}\left(X_{0}, S_{0}\right)+\int_{0}^{t}\left(L V_{*}\right)\left(X_{r}, S_{r}\right) d r \\
& +\int_{0}^{t} \frac{\partial V_{*}}{\partial s}\left(X_{r}, S_{r}\right) d S_{r}+\int_{0}^{t} \frac{\partial V_{*}}{\partial x}\left(X_{r}, S_{r}\right) d B_{r}
\end{aligned}
$$

where (see (1.1) and (2.6))

$$
\left(L V_{*}\right)(x, s)=\frac{1}{2}\left(\frac{\alpha-1}{x} \frac{\partial V_{*}}{\partial x}+\frac{\partial^{2} V_{*}}{\partial x^{2}}\right)
$$

and the stochastic integral with respect to $d B_{r}$ is a local martingale. For the function $V_{*}(x, s)$, given in $(1.13)$,

$$
\left(L V_{*}\right)(x, s)=c, \quad(x, s) \in C_{*}^{1} \cup C_{*}^{2},
$$

and

$$
\left(L V_{*}\right)(x, s)=0, \quad(x, s) \in D_{*} \cap\{(x, s): x>0\} .
$$

Therefore, for each finite Markov time $\tau$ we find, from (3.35)-(3.37), that

$$
V_{*}\left(X_{\tau}, S_{\tau}\right) \leq V_{*}\left(X_{0}, S_{0}\right)+c \tau+\int_{0}^{\tau} \frac{\partial V_{*}}{\partial s}\left(X_{r}, S_{r}\right) d S_{r}+\int_{0}^{\tau} \frac{\partial V_{*}}{\partial x}\left(X_{r}, S_{r}\right) d B_{r}
$$

Note that in this case

$$
\int_{0}^{\tau} \frac{\partial V_{*}}{\partial x}\left(X_{r}, S_{r}\right) d B_{r}=0
$$

since if $X_{r}=S_{r}$, then $\frac{\partial V_{*}}{\partial x}\left(X_{r}, S_{r}\right)=0$ (see (3.18)); if $X_{r}<S_{r}$, then for all $t \geq r$ such that $t<\inf \left\{n \geq r: X_{n}=S_{r}\right\}$ the process $S$ does not change and thus the increment $S_{t}-S_{r}=0$.

Hence from (3.38) and (3.39) it follows that, for each stopping time with $\mathbf{E}_{x, s} \tau<$ $\infty$

$$
\mathbf{E}_{x, s} V_{*}\left(X_{\tau}, S_{\tau}\right)-c \mathbf{E}_{x, s} \tau \leq V_{*}(x, s)+\mathbf{E}_{x, s} \int_{0}^{\tau} \frac{\partial V_{*}}{\partial x}\left(X_{r}, S_{r}\right) d B_{r}
$$

Let us consider the stochastic integral in the right part of this inequality. We have

$$
\begin{aligned}
\int_{0}^{\tau} \frac{\partial V_{*}}{\partial x}\left(X_{r}, S_{r}\right) d B_{r}= & \int_{0}^{\tau} \frac{\partial V_{*}}{\partial x}\left(X_{r}, S_{r}\right) I\left(\left(X_{r}, S_{r}\right) \in C_{*}^{1} \cup C_{*}^{2}\right) d B_{r} \\
= & \frac{2 c}{\alpha} \int_{0}^{\tau} X_{r} I\left(\left(X_{r}, S_{r}\right) \in C_{*}^{1} \cup C_{*}^{2}\right) d B_{r} \\
& -\frac{2 c}{\alpha} \int_{0}^{\tau} X_{r}^{1-\alpha} g_{*}^{\alpha}\left(S_{r}\right) I\left(\left(X_{r}, S_{r}\right) \in C_{*}^{1}\right) d B_{r}
\end{aligned}
$$


If $\left(X_{r}, S_{r}\right) \in C_{*}^{2}$, then $X_{r} \leq S_{*}$ and, therefore, for each $\tau$ with $\mathbf{E}_{x, s} \tau<\infty$ (even if $\mathbf{E}_{x, s} \sqrt{\tau}<\infty ;$ see [20], [31])

$$
\mathbf{E}_{x, s} \int_{0}^{\tau} X_{r} I\left(\left(X_{r}, S_{r}\right) \in C_{*}^{2}\right) d B_{r}=0
$$

In the same way, if $\left(X_{r}, S_{r}\right) \in C_{*}^{1}$, then $g\left(S_{r}\right) \leq X_{r} \leq S_{r}$ and thus $X_{r}^{1-\alpha} g_{*}^{\alpha}\left(S_{r}\right) \leq$ $X_{r} \leq S_{r}$. Therefore, if one can prove that for each $\tau$ with $\mathbf{E}_{x, s} \tau<\infty$ the inequality

$$
\mathbf{E}_{x, s}\left(\int_{0}^{\tau} S_{r}^{2} d r\right)^{1 / 2}<\infty
$$

holds, then from the Burkholder-Davis-Gundy inequality ([20], [31]), for local martingales, stopped at the time $\tau$, we will have the expression

$$
\begin{aligned}
\mathbf{E}_{x, s} & \int_{0}^{\tau} X_{r} I\left(\left(X_{r}, S_{r}\right) \in C_{*}^{1}\right) d B_{r} \\
& +\mathbf{E}_{x, s} \int_{0}^{\tau} X_{r}^{1-\alpha} g_{*}^{\alpha}\left(S_{r}\right) I\left(\left(X_{r}, S_{r}\right) \in C_{*}^{1}\right) d B_{r}=0
\end{aligned}
$$

which together with (3.42) proves that

$$
\mathbf{E}_{x, s} \int_{0}^{\tau} \frac{\partial V_{*}}{\partial x}\left(X_{r}, S_{r}\right) d B_{r}=0
$$

To prove (3.43) note that

$$
\mathbf{E}_{x, s}\left(\int_{0}^{\tau} S_{r}^{2} d r\right)^{1 / 2} \leq \mathbf{E}_{x, s}\left(S_{\tau}^{2} \cdot \tau\right)^{1 / 2} \leq\left(\mathbf{E}_{x, s} S_{\tau}^{2} \cdot \mathbf{E}_{x, s} \tau\right)^{1 / 2}
$$

where

$$
\mathbf{E}_{x, s} S_{\tau}^{2}=\mathbf{E}\left(s^{2} \wedge \max _{r \leq \tau}\left|X_{r}(x)\right|^{2}\right) \leq s^{2}+\mathbf{E} \max _{r \leq \tau}\left|X_{r}(x)\right|^{2}
$$

If $\alpha \geq 1$ and is an integer $(\alpha=d)$, then for the estimation of $\operatorname{Emax}_{r \leq \tau}\left|X_{r}(x)\right|^{2}$ one can use (2.3) which together with the Doob inequality $\left(\mathbf{E}_{\sup _{t \leq \tau}}\left|B_{t}^{1}\right|^{2} \leq\right.$ $\left.4 \mathbf{E}\left(B_{\tau}^{1}\right)^{2} \leq 4 \mathbf{E} \tau\right)$ leads to the inequality

$$
\mathbf{E} \max _{r \leq \tau}\left|X_{r}(x)\right|^{2} \leq x^{2}+4 \alpha \mathbf{E} \tau
$$

If $\alpha$ is an arbitrary non-negative number and $X \in \operatorname{Bes}^{\alpha}(x)$, then from the comparison theorem for the solution of (2.1) we obtain (see, for example, [20, Chap. IX, $\S 3])$ the validity of the estimate (3.46) with $d=[\alpha]+1$.

Thus (3.43) has been established. Hence we have inequality (3.44), which together with (3.42) proves property (3.45). By virtue of (3.40) this proves (3.5), i.e., the property $\left(A_{1}\right)$. Let us show that for $\tau_{*}$ given in Theorem 1, we have (3.6), i.e., property $\left(A_{2}\right)$. By analyzing the preceding proof of $\left(A_{1}\right)$ we notice that the inequality in (3.38) arose because, for each $\tau$,

$$
\int_{0}^{\tau}\left(L V_{*}\right)\left(X_{r}, S_{r}\right) d r \leq c \tau
$$


However, for $\tau=\tau_{*}$ we have equality, because $\left(L V_{*}\right)\left(X_{r}, S_{r}\right)=c$ for $r \leq \tau_{*}$ in accordance with (3.36), if $\left(X_{0}, S_{0}\right) \in C_{*}^{1} \cup C_{*}^{2}$. Together with (3.39) this leads to the equality

$$
V_{*}\left(X_{\tau_{*}}, S_{\tau_{*}}\right)=V_{*}\left(X_{0}, S_{0}\right)+c \tau_{*}+\int_{0}^{\tau_{*}} \frac{\partial V_{*}}{\partial x}\left(X_{r}, S_{r}\right) d B_{r}
$$

which holds for all $\left(X_{0}, S_{0}\right)$ (in case $\left(X_{0}, S_{0}\right) \in D_{*}$ this equality is obvious). Thus, all that is left to prove for $\left(A_{2}\right)$ is to establish that $\mathbf{E}_{x, s} \tau_{*}<\infty,(x, s) \in C_{*}^{1} \cup C_{*}^{2}$. Since, as was shown above (see (3.33)), $\mathbf{E}_{x, s} \sigma_{*}=\left(s_{x}^{2}-x^{2}\right) / \alpha<\infty$ for $(x, s) \in C_{*}^{2}$, it is sufficient to prove that $\mathbf{E}_{x, s} \tau_{*}<\infty$ for $(x, s) \in C_{*}^{1}$.

In the domain $C_{*}^{1}$, where $g_{*}(s)<x \leq s$, the function $m(x, s)=\mathbf{E}_{x, s} \tau^{*}$ is, for each fixed $s$, the solution of the differential equation

$$
\operatorname{Lm}(x, s)=-1
$$

(see Theorem 13.16 in [29]), satisfying the boundary condition $\lim _{x \downarrow g_{*}(s)} m(x, s)=0$ and the condition of normal "reflection" from the diagonal

$$
\left.\frac{\partial m(x, s)}{\partial s}\right|_{x=s}=0
$$

(see, for example, [21, Chap. V, (6.7)]).

Taking into account these facts we readily find that

$$
m(x, s)=-\frac{1}{\alpha}\left(x^{2}-g_{*}^{2}(s)\right)-\frac{2 g_{*}^{2}(s)}{\alpha(\alpha-2)}\left[\left(\frac{g_{*}(s)}{x}\right)^{\alpha-2}-1\right], \quad(x, s) \in C_{*}^{1},
$$

which, in particular, yields the required finiteness of $\mathbf{E}_{x, s} \tau_{*}$.

Thus the optimality of $\tau_{*}$ is established in case $\alpha>1$.

\section{The case when $\alpha=1$. Optimal stopping of the Brownian motion with a drift and instantaneous reflection}

1. In case $\alpha=1$, the Bessel process $X=\left(X_{t}(x)\right)_{t \geq 0}, x \geq 0$, is a Markov process with the infinitesimal operator

$$
L=\frac{1}{2} \frac{d^{2}}{d x^{2}}, \quad x>0
$$

and the boundary point $x=\{0\}$, which is a reflecting boundary. It is well known $([20$, p. 81$])$ that such process can be realized as the modulus of a Brownian motion $\left(B_{0}=0\right)$ :

$$
X_{t}(x)=\left|x+B_{t}\right|
$$

Using Tanaka's formula ([20, Chap. VI, §1]) we find that

$$
\left|x+B_{t}\right|=x+\int_{0}^{t} \operatorname{sign}\left(x+B_{s}\right) d B_{s}+L_{t}^{0}
$$

where $L^{0}=\left(L_{t}^{0}\right)_{t \geq 0}$ is a continuous increasing process (local time of the process $\left(x+B_{t}\right)_{t \geq 0}$ at zero), and $\operatorname{sign} x=1$ for $x>0$ and $\operatorname{sign} x=-1$ for $x \leq 0$. Property 
(4.3) can be reformulated saying that the (non-negative) process $X(x)=\left(X_{t}(x)\right)_{t \geq 0}$ is the solution of the stochastic differential equation with reflection,

$$
d X_{t}=d \beta_{t}+d L_{t}^{0}(X), \quad X_{0}(x)=x
$$

where $\beta=\left(\beta_{t}\right)_{t \geq 0}$ is the Brownian motion process, and $L^{0}=\left(L_{t}^{0}(X)\right)_{t \geq 0}$ a continuous increasing process with $L_{0}^{0}(X)=0$ and

$$
\int_{0}^{\infty} X_{s} d L_{s}^{0}(X)=0
$$

(It would be more correct to say that the process $X(x)$ is the first component of "the process with reflection $\left(X, L^{0}(X)\right)$ ", satisfying equations (4.4) and (4.5)); see [32], [20, Chap. IX] or [21, Chap. V]).

Below we shall not only prove Theorems 1 and 2 for the given case $\alpha=1$, but also examine a somewhat more common case, considering the process $X(x)=\left(X_{t}(x)\right)_{t \geq 0}$, which is the solution of the stochastic differential equation

$$
d X_{t}=\mu d t+d \beta_{t}+d L_{t}^{0}(X), \quad X_{0}(x)=x \geq 0,
$$

where $L^{0}(X)$ satisfies condition (4.5). (If $X_{0}(x)=x>0$, then the process $X$ behaves "locally" as the Brownian motion $\beta_{t}$ with the drift $\mu t$.) Let us assume, as above, that $S_{t}=S_{t}(x, s)$, where

$$
S_{t}(x, s)=\max \left\{s, \sup _{r \leq t} X_{r}(x)\right\}
$$

and let

$$
V_{\mu}(x, s)=\sup _{\tau} \mathbf{E}_{x, s}\left[S_{\tau}-c \tau\right]
$$

THEOREM 3. Let $\mu<c$ and

$$
K_{\mu}=-\frac{1}{2 \mu} \log \left(1-\frac{\mu}{c}\right) .
$$

In "problem (4.8)" the optimal stopping time $\tau_{\mu}$ exists and can be taken in the form

$$
\tau_{\mu}=\inf \left\{t: S_{t}-X_{t} \geq K_{\mu}\right\}
$$

Thus

$$
V_{\mu}(x, s)= \begin{cases}s, & (x, s) \in D_{\mu} \\ s+\frac{c}{2 \mu^{2}}\left[e^{2 \mu\left(g_{\mu}(s)-x\right)}-2 \mu\left(g_{\mu}(s)-x\right)-1\right], & (x, s) \in C_{\mu}^{1} \\ K_{\mu}+\frac{c}{\mu} x+\frac{c}{2 \mu^{2}}\left[e^{-2 \mu x}-1\right], & (x, s) \in C_{\mu}^{2}\end{cases}
$$

where $g_{\mu}(s)=s-K_{\mu}, D_{\mu}=\left\{(x, s): s \geq x+K_{\mu}\right\}, C_{\mu}^{1}=\left\{(x, s): s-K_{\mu}<x \leq s, s>\right.$ $\left.K_{\mu}\right\}, C_{\mu}^{2}=\left\{(x, s): 0 \leq x \leq s \leq K_{\mu}\right\}$.

Corollary. For $\mu=0$ the value $V_{\mu}(x, s)$ coincides with the function $V_{*}^{1}(x, s)$, given by formula (1.13) for $\alpha=1$. In case $(x, s)=(0,0)$,

$$
V_{\mu}(0,0)=K_{\mu}=-\frac{1}{2 \mu} \log \left(1-\frac{\mu}{c}\right) \rightarrow \frac{1}{2 c}, \quad \mu \rightarrow 0 .
$$


Preliminary consideration. The infinitesimal operator $L$ of the process $X(x)$, satisfying the equation (4.6) has the form

$$
L=\mu \frac{d}{d x}+\frac{1}{2} \frac{d^{2}}{d x^{2}}
$$

Using the same scheme of establishing the value $V(x, s)$ as in the case $\mu=0, \alpha=1$, we find (compare with (3.10)), that (under the a priori assumption of "smoothness" of the value) $V_{\mu}(x, s)$ satisfies the equation

$$
\mu \frac{\partial V(x, s)}{\partial x}+\frac{1}{2} \frac{\partial^{2} V(x, s)}{\partial x^{2}}=c
$$

the common solution of which (for each fixed $s$ ) has the following form:

$$
V(x, s)=a(s)+b(s) e^{-2 \mu x}+\frac{c}{\mu} x .
$$

We shall find the independent constants $a(s), b(s)$ and the boundary $g=g(s)$ (compare with the case $\mu=0, \alpha=1$, considering $\S 3$ ) from the additional conditions

$$
V(g(s), s)=s, \quad \frac{\partial V}{\partial x}(g(s), s)=0, \quad \frac{\partial V}{\partial s}(s, s)=0
$$

and the assumption of "smoothness" of the boundary $g=g(s)$ and the value. From the second condition and (4.14) we find that

$$
\frac{c}{\mu}=2 \mu b(s) e^{-2 \mu g(s)} .
$$

From here and from the first condition in (4.15) we see that

$$
a(s)+\frac{c}{2 \mu^{2}}+\frac{c}{\mu} g(s)=s .
$$

Finally the third condition in (4.15) gives

$$
b^{\prime}(s)=-a^{\prime}(s) e^{2 \mu s} .
$$

From (4.16),

$$
b^{\prime}(s) e^{-2 \mu g(s)}-\frac{c}{\mu} g^{\prime}(s)=0
$$

and from (4.17),

$$
a^{\prime}(s)+\frac{c}{\mu} g^{\prime}(s)=1
$$

Formulas (4.18)-(4.20) give the equation for $g=g(s)$ :

$$
\frac{c}{\mu} g^{\prime}\left[1-e^{2 \mu(g-s)}\right]=1
$$

It should be noted that as $\mu \rightarrow 0$ this equation takes the form of the equation

$$
2 c g^{\prime}=\frac{1}{s-g}
$$


from Theorem 1 in $\S 1$ (see (1.10)).

We see directly that equation (4.21) has (in the class of solutions $g=g(s)$, such that $\left.\lim _{s \rightarrow \infty}(g(s) / s)=1\right)$ the solution $g(s)=s-K$, where the constant $K$ can be readily found by substituting $g(s)=s-K$ in (4.21):

$$
\frac{c}{\mu}\left[1-e^{-2 \mu K}\right]=1
$$

Consequently, $K=K_{\mu}=-\log (1-\mu / c) /(2 \mu)$. Finally, from (4.17) and (4.18) we get

$$
\begin{gathered}
a(s)=s-\frac{c}{2 \mu^{2}}-\frac{c}{\mu}\left(s-K_{\mu}\right), \\
b(s)=\frac{c}{2 \mu^{2}} e^{2 \mu\left(s-K_{\mu}\right)} .
\end{gathered}
$$

From (4.23), (4.24), and (4.14) we obtain the representation for $V_{\mu}(x, s)$ in the domain $C_{\mu}^{1}$. As to the representation for $V_{\mu}(x, s)$ in the domain $C_{\mu}^{2}$, one must note first that in this case (for $(x, s) \in C_{\mu}^{2}$ )

$$
V_{\mu}(x, s)=V_{\mu}\left(K_{\mu}, K_{\mu}\right)-c \mathbf{E}_{x, s} \sigma_{\mu}
$$

where (see Fig. 5)

$$
\sigma_{\mu}=\inf \left\{t:\left(X_{t}, S_{t}\right)=\left(K_{\mu}, K_{\mu}\right)\right\} .
$$

Thus, since $\left(K_{\mu}, K_{\mu}\right) \in C_{\mu}^{1}$ and

$$
V_{\mu}\left(K_{\mu}, K_{\mu}\right)=K_{\mu}+\frac{C}{2 \mu^{2}}\left[e^{-2 \mu K_{\mu}}+2 \mu K_{\mu}-1\right],
$$

it suffices to find $\widetilde{M}_{\mu}(x, s)=\mathbf{E}_{x, s} \sigma_{\mu}$. In the domain $C_{\mu}^{2}$, the function $\widetilde{M}_{\mu}(x, s)$ satisfies the equation

$$
\mu \frac{\partial M}{\partial x}(x, s)+\frac{1}{2} \frac{\partial^{2} M}{\partial x^{2}}(x, s)=-1
$$

for $0<x<s$ with "reflection" conditions

$$
\left.\frac{\partial M}{\partial x}\right|_{x=0}=0,\left.\quad \frac{\partial M}{\partial s}\right|_{x=s}=0
$$

and $M\left(K_{\mu}, K_{\mu}\right)=0$. The common solution of the equation (4.27) has (for $\mu \neq 0$ ) the following form (compare with (4.14) at $c=-1$ ):

$$
M(x, s)=a(s)+b(s) e^{-2 \mu x}-\frac{1}{\mu x} .
$$

Taking into account the above-mentioned additional conditions, we can readily find that

$$
\widetilde{M}_{\mu}(x, s)=\frac{1}{2 \mu^{2}}\left[e^{-2 \mu K_{\mu}}-e^{-2 \mu x}\right]+\frac{1}{\mu}\left(K_{\mu}-x\right) .
$$

Using this expression and (4.26) we derive from (4.25) the representation for $V_{\mu}(x, s)$ in the domain $C_{\mu}^{2}$ (see (4.11)). 
2. After these preliminary considerations suggesting the possible optimal stopping time and the structure of the value, we proceed to prove Theorem 3 using the same scheme as in Section 5 in $\S 3$.

Proof of Theorem 3. Bearing in mind representation (4.6) and taking into account the possibility of using Itô's formula for $V_{\mu}(x, s)$ (see [20, Chap. IV, §3]) we obtain

$$
\begin{aligned}
V_{\mu}\left(X_{t}, S_{t}\right)= & V_{\mu}\left(X_{0}, S_{0}\right)+\int_{0}^{t} \frac{\partial V_{\mu}}{\partial x}\left(x_{r}, S_{r}\right) d X_{r}+\int_{0}^{t} \frac{\partial V_{\mu}}{\partial s}\left(X_{r}, S_{r}\right) d S_{r}+\frac{1}{2} \int_{0}^{t} \frac{\partial^{2} V_{\mu}}{\partial x^{2}} d r \\
= & V_{\mu}\left(X_{0}, S_{0}\right)+\int_{0}^{t}\left(\mu \frac{\partial V_{\mu}}{\partial x}+\frac{1}{2} \frac{\partial^{2} V_{\mu}}{\partial x^{2}}\right) d r+\int_{0}^{t} \frac{\partial V_{\mu}}{\partial x}\left(X_{r}, S_{r}\right) d \beta_{r} \\
& +\int_{0}^{t} \frac{\partial V_{\mu}}{\partial x}\left(X_{r}, S_{r}\right) d L_{r}^{0}(X)+\int_{0}^{t} \frac{\partial V_{\mu}}{\partial s}\left(X_{r}, S_{r}\right) d S_{r} .
\end{aligned}
$$

The last integral is equal to zero since $S$ "increases" only on the set $\left\{r: X_{r}=S_{r}\right\}$, but $\frac{\partial V_{\mu}}{\partial s}(s, s)=0$. Similarly the last but one integral is equal to zero since $L^{0}(X)$ increases on the set $\left\{r: X_{r}=0\right\}$, and $\frac{\partial V_{\mu}}{\partial x}(0, s)=0$. Note that $L V_{\mu}(x, s) \leq c$ for all $(x, s)$ (in the domain $C_{\mu}^{1} \cup C_{\mu}^{2}, L V_{\mu}=c$, but in the domain $D_{\mu}, L V_{\mu}=0$ ). Therefore, for any Markov finite time $\tau$ it follows from (4.29) that

$$
V_{\mu}\left(X_{\tau}, S_{\tau}\right) \leq V_{\mu}\left(X_{0}, S_{0}\right)+c \tau+\int_{0}^{\tau} \frac{\partial V_{\mu}}{\partial x}\left(X_{r}, S_{r}\right) d \beta_{r}
$$

and then (under the assumption $\mathbf{E}_{x, s} \tau<\infty$ )

$$
\mathbf{E}_{x, s} V_{\mu}\left(X_{\tau}, S_{\tau}\right)-c \mathbf{E}_{x, s} \tau \leq V_{\mu}(x, s)+\mathbf{E}_{x, s} \int_{0}^{\tau} \frac{\partial V_{\mu}}{\partial x}\left(X_{r}, S_{r}\right) d \beta_{r}
$$

Thus in order to prove the property $\left(A_{1}\right)$ (see (3.5)) it is sufficient to show that

$$
\mathbf{E}_{x, s} \int_{0}^{\tau} \frac{\partial V_{\mu}}{\partial x}\left(X_{r}, S_{r}\right) d \beta_{r}=0
$$

or, equivalently, that

$$
\begin{aligned}
\mathbf{E}_{x, s} & \int_{0}^{\tau} \frac{c}{\mu}\left[1-e^{2 \mu\left(S_{r}-X_{r}-K_{\mu}\right)}\right] I\left(\left(X_{r}, S_{r}\right) \in C_{\mu}^{1}\right) d \beta_{r} \\
& +\mathbf{E}_{x, s} \int_{0}^{\tau} \frac{c}{\mu}\left[1-e^{-2 \mu X_{r}}\right] I\left(\left(X_{r}, S_{r}\right) \in C_{\mu}^{2}\right) d \beta_{r}=0 .
\end{aligned}
$$

In the domain $C_{\mu}^{1}$, we have $-K_{\mu} \leq S_{r}-X_{r}-K_{\mu} \leq 0$. And in the domain $C_{\mu}^{2}$, $0 \leq$ $X_{r} \leq K_{\mu}$. Therefore, for any $\mu \neq 0, \mu<c$, the integrands in the stochastic integrals in (4.33) are bounded and, consequently, the assumption $\mathbf{E}_{x, s} \tau<\infty$ ensures the fulfillment of equality (4.33). The case $\mu=0$ is considered in a similar way.

Thus, property $\left(A_{1}\right)$ holds for all $\mu<c$.

Now let us consider $\tau_{\mu}=\inf \left\{t: S_{t}-X_{t} \geq K_{\mu}\right\}$. As in $\S 3$, to see that property $\left(A_{2}\right)$ is satisfied for this stopping time it is sufficient to establish that $\mathbf{E}_{x, s} \tau_{\mu}<\infty$. This follows directly from the following representation for $M_{\mu}(x, s)=\mathbf{E}_{x, s} \tau_{\mu}$ :

$$
\begin{aligned}
& M_{\mu}(x, s) \\
& = \begin{cases}\frac{1}{\mu}\left(s-x-K_{\mu}\right)+\frac{1}{2 \mu^{2}}\left(e^{2 \mu K_{\mu}}-e^{2 \mu(s-x)}\right), & (x, s) \in C_{\mu}^{1}, \\
-\frac{1}{2 \mu^{2}}\left(e^{-2 \mu x}+2 \mu x+1\right)+\frac{1}{2 \mu^{2}}\left(e^{2 \mu K_{\mu}}+e^{-2 \mu K_{\mu}}\right), & (x, s) \in C_{\mu}^{2},\end{cases}
\end{aligned}
$$


to prove which it is sufficient to note the following. tions

In the domain $C_{\mu}^{1}$, the function $M_{\mu}(x, s)$ satisfies the equation (4.27) with condi-

$$
\left.\frac{\partial M}{\partial s}\right|_{x=s}=0, \quad M\left(s-K_{\mu}, s\right)=0 .
$$

We immediately verify that the first formula in (4.34) gives $M_{\mu}(x, s)$ in the domain $C_{\mu}^{1}$. As for the representation of $M_{\mu}(x, s)$ in the domain $C_{\mu}^{2}$, it follows from

$$
M_{\mu}(x, s)=\widetilde{M}_{\mu}(x, s)+M_{\mu}\left(K_{\mu}, K_{\mu}\right), \quad(x, s) \in C_{\mu}^{2},
$$

where $\widetilde{M}_{\mu}(x, s)$ is determined in $(4.28)$, and $M_{\mu}\left(K_{\mu}, K_{\mu}\right)$ is determined from the first formula in (4.34).

We point out a number of simple corollaries directly following from (4.34):

$$
M_{\mu}(0,0)=\frac{1}{2 \mu^{2}}\left[e^{2 \mu K_{\mu}}+e^{-2 \mu K_{\mu}}-2\right] .
$$

Thus, assuming $\mu \rightarrow 0$, we see that

$$
M_{0}(0,0)=\mathbf{E}_{0,0} \tau^{*}=\frac{1}{2 c^{2}}
$$

where $\tau^{*}=\inf \left\{t: S_{t}-X_{t} \geq 1(2 c)\right\}$. As $\mu \rightarrow 0,(4.34)$ also yields

$$
M_{0}(x, s)= \begin{cases}1 /\left(4 c^{2}\right)-(x-s)^{2}, & (x, s) \in C_{1}^{*} \\ 1 /\left(2 c^{2}\right)-x^{2}, & (x, s) \in C_{2}^{*}\end{cases}
$$

Thus, properties $\left(A_{1}\right)$ and $\left(A_{2}\right)$ are established, which proves Theorem 3.

3. The above-mentioned method of formulating the optimal stopping rules for Brownian motion with drift and reflection (see equation (4.6)) can be used in the case of Brownian motion (with drift) on the whole straight line $\mathbf{R}$.

Namely, let $X(x)=\left(X_{t}(x)\right)_{t \geq 0}$ be a process such that

$$
d X_{t}=\mu d t+d \beta_{t}, \quad X_{0}=x,
$$

where $\beta=\left(\beta_{t}\right)_{t \geq 0}$ is a process of Brownian motion and $-\infty<x<\infty$. Set $S_{t}(x, s)=$ $s \vee \max _{r \leq t} X_{r}(x)$ assume that $s \geq 0$, and let

$$
W_{\mu}(x, s)=\sup _{\sigma} \mathbf{E}_{x, s}\left[S_{\sigma}(x, s)-c \sigma\right] .
$$

THEOREM 4. Let $\mu<c$ and

$$
K_{\mu}=-\frac{1}{2 \mu} \log \left(1-\frac{\mu}{c}\right) .
$$

The optimal stopping time $\sigma_{\mu}$ in problem (4.39) exists and can be taken in the form

$$
\sigma_{\mu}=\inf \left\{t: S_{t}-X_{t} \geq K_{\mu}\right\} .
$$

Moreover,

$$
W_{\mu}(x, s)= \begin{cases}s, & s-x \geq K_{\mu} \\ s+\frac{c}{2 \mu^{2}}\left[e^{2 \mu\left(g_{\mu}(s)-x\right)}-2 \mu\left(g_{\mu}(s)-x\right)-1\right], & s-x \leq K_{\mu}\end{cases}
$$


where $g_{\mu}(s)=s-K_{\mu}$.

The proof is basically the same as that of Theorem 3 and, therefore, is omitted.

4. Let us compare the results of Theorems 3 and 4 in case $\mu=0$. Then, from $(4.11)$

$$
V_{0}(x, s)= \begin{cases}s, & s-x \geq 1 /(2 c) \\ c(x-s)^{2}+x+1 /(4 c), & s \geq 1 /(2 c), s-x \leq 1 /(2 c) \\ c x^{2}+1 /(2 c), & 0 \leq s \leq 1 /(2 c)\end{cases}
$$

and from (4.40)

$$
W_{0}(x, s)= \begin{cases}s, & s-x \geq 1 /(2 c), \\ c(x-s)^{2}+x+1 /(4 c), & s-x<1 /(2 c) .\end{cases}
$$

In particular,

$$
V_{0}(0,0)=\frac{1}{2 c}, \quad W_{0}(0,0)=\frac{1}{4 c}
$$

We have also

$$
\mathbf{E}_{0,0} \tau_{0}=\frac{1}{2 c^{2}}, \quad \mathbf{E}_{0,0} \sigma_{0}=\frac{1}{4 c^{2}}
$$

(The first formula follows from (4.37), the second from $\mathbf{E}_{x, s} \sigma_{0}=1 /\left(4 c^{2}\right)-(x-s)^{2}$.) Thus

$$
\sup _{\tau} \mathbf{E}\left[\max _{r \leq \tau}\left|B_{r}\right|-c \tau\right]=\frac{1}{2 c}
$$

and

$$
\sup _{\sigma} \mathbf{E}\left[\max _{r \leq \sigma} B_{r}-c \sigma\right]=\frac{1}{4 c}
$$

\section{The case when $\alpha<1$}

1. This case should be divided into two: $0<\alpha<1$ and $\alpha \leq 0$. This is connected with the fact that in the first case the domain $C_{*}$ consists of two subdomains, $C_{*}=C_{*}^{1} \cup C_{*}^{2}$, and the point $s_{*}, 0<s_{*}<1 /(2 c)$, and in the second case, $\alpha \leq 0$, the point $s_{*}=0$ and the domain $C_{*}^{2}=\varnothing$.

As we have already mentioned in $\S 2$ in case $0<\alpha<1$ the process $X \in \operatorname{Bes}^{\alpha}(x)$ is not a semimartingale since it is a Dirichlet process. This is connected with the fact that in order to apply the traditional "Itô formula" for the nonsemimartingale $(X, S)$ one must give a special proof which is derived in [3] and [11].

We point out, however, that the functions $V(x, s)=V_{*}^{\alpha}(x, s)$, defined in (1.13), actually depend on $x$ through $y=x^{2}$ and, therefore, one can apply to the process $U\left(Y_{t}, S_{t}\right)$, where $U(y, s)=V(x, s), y=x^{2}$, the traditional semimartingale Itô formula (since $Y$ is a semimartingale and $U(y, s)$ is a sufficient by smooth function):

$$
\begin{aligned}
U\left(Y_{t}, S_{t}\right)= & U\left(Y_{0}, S_{0}\right)+\int_{0}^{t} \frac{\partial U}{\partial y}\left(Y_{r}, S_{r}\right) d Y_{r} \\
& +\int_{0}^{t} \frac{\partial U}{\partial s}\left(Y_{r}, S_{r}\right) d S_{r}+\int_{0}^{t} \frac{1}{2} \frac{\partial^{2} U}{\partial y^{2}}\left(Y_{r}, S_{r}\right)\left(4 Y_{r}\right) d r
\end{aligned}
$$




$$
\begin{aligned}
= & U\left(Y_{0}, S_{0}\right)+\int_{0}^{t}\left[\alpha \frac{\partial U}{\partial y}\left(Y_{r}, S_{r}\right)+2 Y_{r} \frac{\partial^{2} U}{\partial y^{2}}\left(Y_{r}, S_{r}\right)\right] d r \\
& +\int_{0}^{t} \frac{\partial U}{\partial s}\left(Y_{r}, S_{r}\right)+\int_{0}^{t} 2 \frac{\partial U}{\partial y}\left(Y_{r}, S_{r}\right) \sqrt{Y_{r}} d B_{r} .
\end{aligned}
$$

Remark. In case $\alpha \geq 1$ one could also operate with the process $Y$ and not with the process $X$. However, in our case we preferred to deal directly with the original process $X$ which corresponds better to the sense of the problem under consideration.

The expression in square brackets in equation (5.1) is equal to zero in the domain $D_{*}$ and is equal to $c$ in the domain $C_{*}$. As in the case $\alpha \geq 1$ the integral with respect to $d S_{r}$ is equal to zero and in order to prove property $\left(A_{1}\right)$ one must show that if $\mathbf{E}_{y, s} \tau<\infty$, then

$$
\mathbf{E}_{y, s} \int_{0}^{\tau} \frac{\partial U}{\partial y}\left(Y_{r}, S_{r}\right) \sqrt{Y_{r}} d B_{r}=0 .
$$

In turn, for this it is sufficient to just show that

$$
\mathbf{E}_{y, s}\left[\int_{0}^{\tau}\left(\frac{\partial U}{\partial y}\left(Y_{r}, S_{r}\right)\right)^{2} Y_{r} d r\right]^{1 / 2}<\infty
$$

Since in the domain $C_{*}^{2} \frac{\partial U}{\partial y}\left(Y_{r}, S_{r}\right)=c / \alpha$,

$$
\int_{0}^{\tau}\left(\frac{\partial U}{\partial y}\left(Y_{r}, S_{r}\right)\right)^{2} Y_{r} I\left(\sqrt{Y_{r}} \leq S_{r} \leq s_{*}(\alpha)\right) d r \leq\left(\frac{c s_{*}(\alpha)}{\alpha}\right)^{2} \tau
$$

and, therefore,

$$
\mathbf{E}_{y, s}\left[\int_{0}^{\tau}\left(\frac{\partial U}{\partial y}\left(Y_{r}, S_{r}\right)\right)^{2} Y_{r} I\left(\sqrt{Y_{r}} \leq S_{r} \leq s_{*}(\alpha)\right)\right]^{1 / 2}<\infty
$$

In the domain $C_{*}^{1}=\left\{(x, s): g_{*}(s)<x \leq s, s_{*}<s\right\}$,

$$
\frac{\partial U}{\partial y}\left(Y_{r}, S_{r}\right)=\frac{c}{\alpha}\left[1-\frac{g_{*}^{\alpha}\left(S_{r}\right)}{X_{r}^{\alpha}}\right]
$$

with $X_{r}=\left(Y_{r}\right)^{1 / 2}$. Therefore, in this case,

$$
\left(\frac{\partial U}{\partial y}\right)^{2} \leq 4\left(\frac{c}{\alpha}\right)^{2}
$$

and to prove (5.3) we must show that

$$
\mathbf{E}_{y, s}\left(\int_{0}^{\tau} Y_{r} d r\right)^{1 / 2}<\infty
$$

for any Markov time $\tau$ with $\mathbf{E}_{x, s} \tau<\infty, x=y^{1 / 2}$.

But

$$
\mathbf{E}_{x, s}\left(\int_{0}^{\tau} Y_{r} d r\right)^{1 / 2} \leq \mathbf{E}_{x, s}\left(S_{\tau}^{2} \cdot \tau\right)^{1 / 2}=\mathbf{E}_{x, s} S_{\tau} \cdot \tau^{1 / 2} \leq\left(\mathbf{E}_{x, s} S_{\tau}^{2} \cdot \mathbf{E}_{x, s} \tau\right)^{1 / 2}
$$

where the finiteness of $\mathbf{E}_{x, s} S_{\tau}^{2}=\mathbf{E}_{x, s}\left(\max _{r \leq \tau} X_{r}\right)^{2}$ for the processes $X \in \operatorname{Bes}^{\alpha}(x), 0<$ $\alpha<1$, follows from the comparison theorem for the processes $Y \in \operatorname{Besq}^{\alpha}(y), 0<\alpha<$ 
1, and $Y \in \operatorname{Besq}^{1}(y)$ because for the last process $\mathbf{E}_{x, s} \max _{r \leq \tau} Y_{r}<\infty$ according to Doob's inequality.

Thus property $\left(A_{1}\right)$ is established.

For the proof of property $\left(A_{2}\right)$, which is carried out exactly in the same way as in the case $\alpha \geq 1$, we only need to determine the finiteness of $m(x, s)=\mathbf{E}_{x, s} \tau^{*}$, which follows actually from the explicit form of $m(x, s)$ in the domains $C_{*}^{1}$ and $C_{*}^{2}$ : if $(x, s) \in C_{*}^{1}$ then $m(x, s)$ is given by formula (3.49), and if $(x, s) \in C_{*}^{2}$, then $m(x, s)=$ $m\left(s_{*}, s_{*}\right)+\mathbf{E}_{x, s} \sigma_{*}$, where $\sigma_{*}=\inf \left\{t:\left(X_{t}, S_{t}\right)=\left(s_{*}, s_{*}\right)\right\}$ and $\mathbf{E}_{x, s} \sigma_{*}=\left(s_{*}^{2}-x^{2}\right) / \alpha$ (compare with (3.33)).

Now let us turn to the case $\alpha \leq 0$. In this case, the point $x=\{0\}$ and the point $y=\{0\}$ are traps for the processes $X \in \operatorname{Bes}^{\alpha}(x)$ and $Y \in \operatorname{Besq}^{\alpha}(y)$. Here the process $Y$, having the stochastic differential (see (2.1)) $d Y_{t}=\alpha d t+2 \sqrt{Y_{t}} d B_{t}$, is a non-negative supermartingale and, consequently, arriving in the zero state it remains in this state. Hence it follows that the set $\{x, s: x=0, s \geq x\}$ is clearly contained in the stopping domain $D_{*}$.

Let $x>0, s>0$, and $y=x^{2}>0$. As in the case $0<\alpha<1$ considered above to prove property $\left(A_{1}\right)$ we must show the validity of inequality (5.3) for any Markov time $\tau$ with $\mathbf{E}_{x, s} \tau<\infty$.

If $\alpha=0$, then, in the domain $C_{*}$,

$$
\frac{\partial U}{\partial y}\left(Y_{r}, S_{r}\right)=\frac{c}{2} \log \left(\frac{Y_{r}}{g^{2}\left(S_{r}\right)}\right)=c \log \frac{X_{r}}{g\left(S_{r}\right)} .
$$

And if $\alpha<0$, then, in the domain $C_{*}^{1}$,

$$
\begin{aligned}
\frac{\partial U}{\partial y}\left(Y_{r}, S_{r}\right) & =\frac{c}{\alpha}\left(1-\frac{g^{\alpha}\left(S_{r}\right)}{Y_{r}^{\alpha / 2}}\right)=\frac{c}{\alpha}\left(1-\left(\frac{g\left(S_{r}\right)}{X_{r}}\right)^{\alpha}\right) \\
& =-\frac{c}{\beta}\left(1-\left(\frac{X_{r}}{g\left(S_{r}\right)}\right)^{\beta}\right)
\end{aligned}
$$

with $\beta=-\alpha>0$.

Note that if the starting point $\left(X_{0}, S_{0}\right)=\left(x_{0}, s_{0}\right)$ with $x_{0}>0, s_{0}>0$, then from equations (1.7) and condition (1.8) one can draw the conclusion that, in the domain $C_{*}$

$$
1 \leq \frac{X_{r}}{g\left(S_{r}\right)} \leq \frac{S_{r}}{g\left(S_{r}\right)}=\frac{s_{0}}{g\left(s_{0}\right)} .
$$

Therefore, in the domain $C_{*},\left(\frac{\partial U}{\partial y}\left(Y_{r}, S_{r}\right)\right)^{2}$ is less than some constant (depending on $\alpha$ and the initial point $x>0, s>0$ ) and, consequently, it follows from (5.3) that it is sufficient just to establish inequality (5.5), the validity of which (again using the comparison theorem for processes $\left.Y \in \operatorname{Besq}^{\alpha}(y)\right)$ is established in the same way as in the case $0<\alpha<1$.

This proves the validity of property $\left(A_{1}\right)$. Property $\left(A_{2}\right)$ follows from the finiteness of $m(x, s)=\mathbf{E}_{x, s} \tau^{*}$, determined by formula (3.49) for $\alpha \neq 0$ and the formula

$$
m(x, s)=-\frac{1}{2}\left[g_{*}^{2}(s)-x^{2}\right]-x^{2} \log \frac{x}{g_{*}(s)}, \quad(x, s) \in C_{*}
$$

for $\alpha=0$. 


\section{On maximal inequalities for the Bessel process} value

1. Let $X \in \operatorname{Bes}^{\alpha}(x), S_{t}=S_{t}(x, s)=s \vee \max _{r \leq t} X_{r}(x)$. We shall consider the

$$
V_{c}^{\alpha}(x, s)=\sup _{\tau} \mathbf{E}_{x, s}\left[S_{\tau}-c \tau\right]
$$

where we have introduced the index $c$ in its notation.

The Bessel processes have the same property of self-similarity as does Brownian motion: for $c>0$,

$$
c^{-1 / 2} X_{c t}\left(c^{1 / 2} x\right) \stackrel{\mathrm{d}}{=} X_{t}(x) \text {. }
$$

(See, for example, [20, Chap. XI, (1.10)]). From this it is easy to show that the value $V_{c}^{\alpha}(x, s)$ has the following property of self-similarity:

$$
c V_{c}^{\alpha}(x, s)=V_{1}^{\alpha}(c x, c s) .
$$

In particular,

$$
c V_{c}^{\alpha}\left(0, \frac{s}{c}\right)=V_{1}^{\alpha}(0, s) .
$$

We shall denote by $s_{c}(\alpha)$ the root of the equation $g_{*}(s)=0$, where $g_{*}=g_{*}(s)$ is given by equation (1.7) with condition (1.8) (for given $\alpha$ and $c$ ). According to Theorem 2,

$$
V_{c}^{\alpha}\left(0, s_{c}(\alpha)\right)=s_{c}(\alpha)
$$

and, in particular,

$$
V_{1}^{\alpha}\left(0, s_{1}(\alpha)\right)=s_{1}(\alpha)
$$

From (6.4),

$$
s_{1}(\alpha)=V_{1}^{\alpha}\left(0, s_{1}(\alpha)\right)=c V_{c}^{\alpha}\left(0, \frac{s_{1}(\alpha)}{c}\right) \geq c s_{c}(\alpha),
$$

since $V_{c}^{\alpha}(0, s) \geq s_{c}(\alpha)$ (see (1.13)) and, in the same way,

$$
s_{c}(\alpha)=V_{c}^{\alpha}\left(0, s_{c}(\alpha)\right)=\frac{1}{c} V_{1}^{\alpha}\left(0, c s_{c}(\alpha)\right) \geq \frac{1}{c} s_{1}(\alpha) .
$$

This yields the following main property of self-similarity for $s_{c}(\alpha)$ :

$$
s_{c}(\alpha)=\frac{1}{c} s_{1}(\alpha)
$$

2. From (1.13),

$$
V_{c}^{\alpha}(0,0)=\sup _{\tau} \mathbf{E}\left[\max _{r \leq \tau} X_{r}-c \tau\right]=s_{c}(\alpha),
$$

where $X_{r}=X_{r}(0)$. Together with (6.7) we conclude from (6.8) that, for each $\tau$,

$$
\mathbf{E} \max _{r \leq \tau} X_{r} \leq \frac{s_{1}(\alpha)}{c}+c \mathbf{E} \tau
$$


On the right side, $c>0$ and it is arbitrary. Therefore,

$$
\mathbf{E} \max _{r \leq \tau} X_{r} \leq \inf _{c>0}\left[\frac{s_{1}(\alpha)}{c}+c \mathbf{E} \tau\right]=\sqrt{4 s_{1}(\alpha) \mathbf{E} \tau}
$$

where the minimum is obtained for $c=\left(s_{1}(\alpha) / \mathbf{E} \tau\right)^{1 / 2}$. Thus, the next theorem is proved.

Theorem 5. Let $X \in \operatorname{Bes}^{\alpha}(0), \alpha \geq 0$. Then,for each stopping time $\tau$,

$$
\mathbf{E} \max _{r \leq \tau} X_{r} \leq \sqrt{4 s_{1}(\alpha) \mathbf{E} \tau}
$$

where $s_{1}(\alpha)$ is the root of the equation $g_{*}(s)=0$ for (the only) function satisfying the equation

$$
\frac{2}{\alpha-2} g^{\prime} g\left[1-\left(\frac{g}{s}\right)^{\alpha-2}\right]=1
$$

such that $\lim _{s \rightarrow \infty}\left(g_{*}(s) / s\right)=1$.

Remark. In case $\alpha \leq 0$ and $X \in \operatorname{Bes}^{\alpha}(0), X_{t} \equiv 0, t \geq 0$, and inequality (6.9) holds in an obvious way, since $s_{1}(\alpha)=0, \alpha \leq 0$.

3. If $\alpha=1$, then $s_{1}(\alpha)=\frac{1}{2}$ and, consequently, we obtain for the Brownian motion $B=\left(B_{t}\right)_{t \geq 0}$ the result of $[10]$ :

$$
\mathbf{E} \max _{r \leq \tau}\left|B_{r}\right| \leq \sqrt{2 \mathbf{E} \tau}
$$

Therefore, if one considers the pair $\left(\mathbf{E} \tau, \mathbf{E} \max _{r \leq \tau}\left|B_{r}\right|\right)$ as the point $(x, y)$ in the first quadrant $\{(x, y): x \geq 0, y \geq 0\}$, one can assert that when $\tau$ runs through the set of admissible Markov times, then the corresponding points $(x, y)$ will belong to the set

$$
\Pi=\{(x, y): x>0,0<y \leq \sqrt{2 x}\} \cup\{(0,0)\} .
$$

Let us show that the inequality in (6.11) is sharp in the sense that if $T$ is an arbitrary fixed number, then there exists a stopping time $\tau=\tau(T)$ such that $\mathbf{E} \tau(T)=$ $T$ and $\mathbf{E} \max _{r \leq \tau(T)}\left|B_{r}\right|=\sqrt{2 T}$. Indeed, let us consider the value

$$
V_{c}^{1}(0,0)=\sup _{\tau} \mathbf{E}\left[\max _{r \leq \tau}\left|B_{r}\right|-c \tau\right]=S_{c}(1)=\frac{1}{2 c} .
$$

If $\tau_{*}(c)=\inf \left\{t: \max _{r \leq \tau}\left|B_{r}\right|-\left|B_{t}\right| \geq 1 /(2 c)\right\}$, then, in accordance with the established optimal property of this time,

$$
\mathbf{E}\left[\max _{r \leq \tau_{*}(c)}\left|B_{r}\right|-c \tau_{*}(c)\right]=\frac{1}{2 c}
$$

From (4.36), $\mathbf{E} \tau_{*}(c)=1 /\left(2 c^{2}\right)$. This means that

$$
\mathbf{E} \max _{r \leq \tau_{*}(c)}\left|B_{r}\right|=\frac{1}{2 c}+\frac{c}{2 c^{2}}=\frac{1}{c} .
$$

Therefore, using $c=\sqrt{1 /(2 T)}$ we obtain $\mathbf{E} \tau_{*}(c)=T$ and $\mathbf{E} \max _{r \leq \tau_{*}(c)}\left|B_{r}\right|=\sqrt{2 T}$, which proves that the inequality in (6.11) is sharp. 
Let us also show that for each given point $(x, y)$ of $\Pi$ there exists a (may be randomized) stopping time $\sigma$ such that $\mathbf{E} \sigma=x$ and $\mathbf{E} \max _{r \leq \sigma}\left|B_{r}\right|=y$. Indeed, if $(x, y)=(0,0)$, then we set $\sigma \equiv 0$. Let $\left(x_{0}, y_{0}\right)$ be some point of the set $\{(x, y): x>$ $0,0<y<\sqrt{2 x}\}$. Let us draw a straight line through $(0,0)$ and $\left(x_{0}, y_{0}\right)$ denoting by $(\tilde{x}, \tilde{y})$ the point of its intersection with the parabola $y=\sqrt{2 x}$. It is clear that

$$
\tilde{x}=\frac{2 x_{0}^{2}}{y_{0}^{2}}, \quad \tilde{y}=\frac{2 x_{0}}{y_{0}} .
$$

The point $(\tilde{x}, \tilde{y})$ lies on the parabola $y=\sqrt{2 x}$ and, as shown above, there exists a Markov time $\tilde{\tau}$ such that $\mathbf{E} \tilde{\tau}=\tilde{x}, \mathbf{E} \max _{r \leq \tilde{\tau}}\left|B_{r}\right|=\tilde{y}$. Let us form a new (randomized, [33]) stopping time

$$
\widehat{\tau}=\left\{\begin{array}{lll}
\tilde{\tau} & \text { with probability } & x_{0} / \tilde{x} \\
0 & \text { with probability } & 1-x_{0} / \tilde{x}
\end{array}\right.
$$

Then $\mathbf{E} \widehat{\tau}=\mathbf{E} \tilde{\tau} \cdot x_{0} / \tilde{x}=x_{0}$ and $\mathbf{E} \max _{r<\widehat{\tau}}\left|B_{r}\right|=\mathbf{E} \max _{r \leq \widehat{\tau}}\left|B_{r}\right| \cdot x_{0} / \tilde{x}=\tilde{y} \cdot x_{0} / \tilde{x}=y_{0}$. Thus one can state that (at least admitting randomized stopping times) each point of $\Pi$ can be reached in the above-mentioned sense.

4. Let us turn to the analogue of (6.11) for $\mathbf{E} \max _{r \leq \sigma} B_{r}$. Using (4.39) and (4.42) one can see that, for each $c>0$,

$$
\mathbf{E} \max _{r \leq \sigma} B_{r} \leq c \mathbf{E} \sigma+\frac{1}{4 c}
$$

and, consequently,

$$
\mathbf{E} \max _{r \leq \sigma} B_{r} \leq \inf _{c>0}\left[c \mathbf{E} \sigma+\frac{1}{4 c}\right] \leq \sqrt{\mathbf{E} \sigma} .
$$

Thus (compare with (6.11)) for each stopping time $\sigma$ we obtain the following result:

$$
\mathbf{E} \max _{r \leq \sigma} B_{r} \leq \sqrt{\mathbf{E} \sigma}
$$

established in [10] as well.

Note that as in (6.11) the inequality in (6.14) is sharp. This follows from the fact that, for $\sigma_{*}(c)=\inf \left\{t \geq 0: \max _{r \leq t} B_{r}-B_{t} \geq 1 /(2 c)\right\}$,

$$
\mathbf{E} \sigma_{*}(c)=\frac{1}{4 c^{2}}, \quad \mathbf{E} \sup _{r \leq \sigma_{*}(c)} B_{r}=\frac{1}{2 c} .
$$

It is also interesting to note that the comparison of formulas (4.45), (4.46), (4.43), and (4.44) show that in "the problem $\mathbf{E} \sup _{\sigma}\left[\max _{r \leq \sigma} B_{r}-c \sigma\right]$ " as compared with "the problem $\mathbf{E} \sup _{\tau}\left[\max _{r \leq \tau}\left|B_{r}\right|-c \tau\right]$ " "everything is twice as bad" which, however, is quite natural in view of the meaning of these problems.

5. Let us again turn to

$$
\tau_{*}(c)=\inf \left\{t: \max _{r \leq t}\left|B_{r}\right|-B_{t} \geq \frac{1}{2 c}\right\}
$$

and

$$
\sigma_{*}(c)=\inf \left\{t: \max _{r \leq t} B_{r}-B_{t} \geq \frac{1}{2 c}\right\}
$$


which are optimal stopping times in the corresponding problems of optimal stopping. As is shown above, $\mathbf{E} \tau_{*}(c)=1 /\left(2 c^{2}\right)$ and $\mathbf{E} \sigma_{*}(c)=1 /(2 c)$. Let us find the probability distribution of $\tau_{*}(c)$ and $\sigma_{*}(c)$.

To this end we note that the (P. Lévy) processes $\max B-B=\left(\max _{r \leq t} B_{r}-B_{t}\right)_{t \geq 0}$ and $|\tilde{B}|=\left(\left|\tilde{B}_{t}\right|\right)_{t \geq 0}$, where $\tilde{B}$ is standard Brownian motion have one and the same distribution:

$$
\operatorname{Law}(\max B-B)=\operatorname{Law}(|\tilde{B}|),
$$

(see [20, Chap. VI, Thm. (2.3)]). Therefore, if $\tilde{\tau}(c)=\inf \left\{t:|\tilde{B}|_{t} \geq 1 /(2 c)\right\}$, then $\operatorname{Law}\left(\sigma_{*}(c)\right)=\operatorname{Law}(\tilde{\tau}(c))$. Since $\mathbf{E} \tilde{\tau}(c)=\mathbf{E} \sigma_{*}(c)<\infty$, we have, for each $\lambda \geq 0$,

$$
\mathbf{E} \exp \left( \pm \lambda \tilde{B}_{\tilde{\tau}(c)}-\frac{\lambda^{2}}{2} \tilde{\tau}(c)\right)=1 .
$$

Hence, supposing $a=1 /(2 c)$, we find $\left(\mathbf{E}(\xi ; A)=\mathbf{E} \xi I_{A}\right)$ :

$$
\mathbf{E}\left[\exp \left\{\lambda a-\frac{\lambda^{2}}{2} \tilde{\tau}(c)\right\} ; \tilde{B}_{\tilde{\tau}(c)}=a\right]+\mathbf{E}\left[\exp \left\{-\lambda a-\frac{\lambda^{2}}{2} \tilde{\tau}(c)\right\} ; \tilde{B}_{\tilde{\tau}(c)}=-a\right]=1
$$

and

$$
\mathbf{E}\left[\exp \left\{-\lambda a-\frac{\lambda^{2}}{2} \tilde{\tau}(c)\right\} ; \tilde{B}_{\tilde{\tau}(c)}=a\right]+\mathbf{E}\left[\exp \left\{\lambda a-\frac{\lambda^{2}}{2} \tilde{\tau}(c)\right\} ; \tilde{B}_{\tilde{\tau}(c)}=a\right]=1 .
$$

Thus $(a=1 /(2 c))$,

$$
\mathbf{E} \exp \left\{-\frac{\lambda^{2}}{2} \sigma_{*}(c)\right\}=\mathbf{E} \exp \left\{-\frac{\lambda^{2}}{2} \tilde{\tau}(c)\right\}=\frac{1}{\cosh \lambda a} .
$$

Similarly, if $\tilde{B}_{0}=b_{0}$, then

$$
\mathbf{E} \exp \left\{-\frac{\lambda^{2}}{2} \tilde{\tau}(c)\right\}=\frac{\cosh \lambda b_{0}}{\cosh \lambda a} .
$$

From the Laplace transform (6.16) we can get a representation (in the form of a series) for the density $p_{\sigma_{*}(c)}(t)$ of the probability distribution $\sigma_{*}(c)$, based on the following approach suggested to us by M. Yor.

Note that

$$
\frac{1}{\cosh \lambda}=\frac{2}{e^{\lambda}\left(1+e^{-2 \lambda}\right)}=\frac{2}{e^{\lambda}} \sum_{n=0}^{\infty}(-1)^{n} e^{-2 n \lambda}=2 \sum_{n=0}^{\infty}(-1)^{n} e^{-(2 n+1) \lambda} .
$$

It is also known that ([20, pp. 101-102]), if $a>0$ and $T_{a}=\inf \left\{t: B_{t}=a\right\}$, then

$$
e^{-\lambda a}=\mathbf{E} \exp \left(-\frac{\lambda^{2}}{2} T_{a}\right)=\int_{0}^{\infty} \frac{a \exp \left\{-a^{2} /(2 t)\right\}}{\sqrt{2 \pi t^{3}}} \exp \left\{-\frac{\lambda^{2}}{2} t\right\} d t
$$

Hence, from (6.17), (6.18), we obtain

$$
\frac{1}{\cosh \lambda}=2 \sum_{n=0}^{\infty}(-1)^{n} \int_{0}^{\infty} \exp \left\{-\frac{\lambda^{2}}{2} t\right\} \frac{(2 n+1) \exp \left\{-(2 n+1)^{2} /(2 t)\right\}}{\sqrt{2 \pi t^{3}}} d t .
$$

From (6.16) it follows that

$$
\mathbf{E} \exp \left\{-\frac{\lambda^{2}}{2}\left((2 c)^{2} \sigma_{*}(c)\right)\right\}=\frac{1}{\cosh \lambda}
$$


Therefore,

$$
\begin{aligned}
\mathbf{E} \exp & \left\{-\frac{\lambda^{2}}{2}(2 c)^{2} \sigma_{*}(c)\right\} \\
= & \int_{0}^{\infty} \exp \left\{-\frac{\lambda^{2}}{2} t\right\} 2 \sum_{n=0}^{\infty}(-1)^{n} \frac{(2 n+1) \exp \left\{-(2 n+1)^{2} /(2 t)\right\}}{\sqrt{2 \pi t^{3}}} d t
\end{aligned}
$$

which gives

$$
p_{(2 c)^{2} \sigma_{*}(c)}(t)=2 \sum_{n=0}^{\infty}(-1)^{n} \frac{(2 n+1) \exp \left\{-(2 n+1)^{2} /(2 t)\right\}}{\sqrt{2 \pi t^{3}}} d t .
$$

To find the distribution of $\tau_{*}(c)$ let us note first of all that $\tau_{*}(c)$ is equal to the sum of the transition time $\tau_{1}(c)$ of the process $(B, \max |B|)$ from the point $(0,0)$ to the point $(1 /(2 c), 1 /(2 c))$ and the exit time $\tau_{2}(c)$ from the point $(1 /(2 c), 1 /(2 c))$ to the domain $D_{*}=\{(x, s): s-x \geq 1 /(2 c), s \geq 1 /(2 c)\}$. Let $X_{t}(x)=x+B_{t}, S_{t}(x, s)=$ $s \wedge \max _{r \leq t}\left|X_{r}(x)\right|, \tau_{1}(c)=\inf \left\{t: X_{t}(x)=a, S_{t}(x, s)=a\right\}, a=1 /(2 c)$. Then $\psi(x, s)=\mathbf{E}_{x, s} e^{-\lambda \tau_{1}(c)}$ is the solution of the equation

$$
\lambda \frac{\partial \psi}{\partial x}=\frac{1}{2} \frac{\partial^{2} \psi}{\partial x^{2}}
$$

with the conditions

$$
\left.\frac{\partial \psi}{\partial x}\right|_{x=0}=0,\left.\quad \frac{\partial \psi}{\partial s}\right|_{x=s}=1
$$

The general solution of equation (6.21) has the form

$$
\psi(x, s)=a(s) \cosh x \sqrt{2 \lambda}+b(s) \sinh x \sqrt{2 \lambda} .
$$

From the first condition in (6.22) we find that $b(s)=0$. From the second condition it follows that $a(s)=$ const. Since $\psi(1 /(2 c), 1 /(2 c))=1$, we obtain for the constant the value $(\cosh \sqrt{2 \lambda} /(2 c))^{-1}$ and thus

$$
\psi(x, s)=\frac{\cosh x \sqrt{2 \lambda}}{\cosh \sqrt{2 \lambda} /(2 c)} .
$$

According to the strong Markov property of the process $(X, S)$ and the fact that $\operatorname{Law}\left(\tau_{2}(c)\right)=\operatorname{Law}\left(\sigma_{*}(c)\right)$ we find for $\tau_{*}(c)=\tau_{1}(c)+\tau_{2}(c)$ that

$\mathbf{E}_{x, s} e^{-\lambda \tau_{*}(c)}=\mathbf{E}_{x, s} e^{-\lambda \tau_{1}(c)} \cdot \mathbf{E}_{0,0} e^{-\lambda \sigma_{*}(c)}=\psi(x, s) \cdot \mathbf{E}_{0,0} e^{-\lambda \sigma_{*}(c)}=\frac{\cosh x \sqrt{2 \lambda}}{(\cosh \sqrt{2 \lambda} /(2 c))^{2}}$.

Therefore, if $x=0, s=0$, then

$$
\mathbf{E} e^{-\lambda \tau_{*}(c)}=\frac{1}{(\cosh \sqrt{2 \lambda} /(2 c))^{2}}
$$

and this means that $(a=1 /(2 c))$

$$
\mathbf{E} \exp \left\{-\frac{\lambda^{2}}{2} \tau_{*}(c)\right\}=\left(\frac{1}{\cosh \lambda a}\right)^{2}=\left(\mathbf{E} \exp \left\{-\frac{\lambda^{2}}{2} \sigma_{*}(c)\right\}\right)^{2} .
$$


Hence it follows that the distribution for $\tau_{*}(c)$ is the convolution of the two distributions for $\sigma_{*}(c)$.

6. Let us consider now the problem of the probability distributions of $S_{\sigma_{*}(c)}=$ $\max _{r \leq \sigma_{*}(c)} B_{r}$ and $S_{\tau_{*}(c)}=\max _{r \leq \tau_{*}(c)}\left|B_{r}\right|$. In view of the strong Markov property of the process $(X, S)$ we conclude that, for any $s_{1} \geq 0, s_{2} \geq 0$,

$$
\mathbf{P}_{0,0}\left(S_{\sigma_{*}(c)} \geq s_{1}+s_{2}\right)=\mathbf{P}_{0,0}\left(S_{\sigma_{*}(c)} \geq s_{1}\right) \cdot \mathbf{P}_{0,0}\left(S_{\sigma_{*}(c)} \geq s_{2}\right) .
$$

This implies that, for some constant $\lambda>0$,

$$
\mathbf{P}_{0,0}\left(S_{\sigma_{*}(c)} \geq s\right)=e^{-\lambda s}, \quad s \geq 0 .
$$

But $\mathbf{E}_{0,0} S_{\sigma_{*}(c)}=1 /(2 c)$ (see (6.15)). Therefore, the constant $\lambda=2 c$ and

$$
\mathbf{P}_{0,0}\left(S_{\sigma_{*}(c)} \geq s\right)=e^{-2 c s}, \quad s \geq 0 .
$$

One can also see easily that $\mathbf{P}_{0,0}\left(S_{\tau_{*}(c)} \geq s+1 /(2 c)\right)=\mathbf{P}_{0,0}\left(S_{\sigma_{*}(c)} \geq s\right)$. Therefore,

$$
\mathbf{P}_{0,0}\left(S_{\tau_{*}(c)} \geq s+\frac{1}{2 c}\right)=e^{-2 c s}, \quad s \geq 0 .
$$

We sum up our results in the form of the following (known to many specialists) statement, being of independent interest.

Theorem 6. Let $B=\left(B_{t}\right)_{t \geq 0}$ be standard Brownian motion,

$$
\sigma_{a}=\inf \left\{t: \max _{r \leq t} B_{r}-B_{t} \geq a\right\}, \quad \tau_{a}=\inf \left\{t: \max _{r \leq t}\left|B_{r}\right|-B_{t} \geq a\right\} .
$$

Then

$$
\begin{aligned}
\mathbf{E} \sigma_{a} & =a^{2}, \quad \mathbf{E} \tau_{a}=2 a^{2} ; \\
\mathbf{E} e^{-\frac{\lambda^{2}}{2} \sigma_{a}} & =\frac{1}{\cosh \lambda a}, \quad \quad \mathbf{E} e^{-\frac{\lambda^{2}}{2} \tau_{a}}=\left(\frac{1}{\cosh \lambda a}\right)^{2}
\end{aligned}
$$

the density of the probability distribution is

$$
p_{\sigma_{a} / a^{2}}(t)=2 \sum_{n=0}^{\infty}(-1)^{n} \frac{(2 n+1) \exp \left\{-(2 n+1)^{2} /(2 t)\right\}}{\sqrt{2 \pi t^{3}}}
$$

the density of the probability distribution $p_{\tau_{a} / a^{2}}$ is the convolution of two densities $p_{\sigma_{a} / a^{2}} ;$ the probabilities are of the form

$$
\mathbf{P}\left(\max _{r \leq \sigma_{a}} B_{r} \geq s\right)=e^{-s / a}, \quad s \geq 0
$$

and

$$
\mathbf{P}\left(\max _{r \leq \tau_{a}}\left|B_{r}\right| \geq s+a\right)=e^{-s / a}, \quad s \geq 0
$$

7. Let $X^{(\alpha)} \in \operatorname{Bes}^{\alpha}(0), \alpha \geq 0$. According to Theorem 5, for each finite Markov time $\tau$,

$$
\mathbf{E} \max _{r \leq \tau} X_{r}^{(\alpha)} \leq \gamma(\alpha) \sqrt{\mathbf{E} \tau}
$$




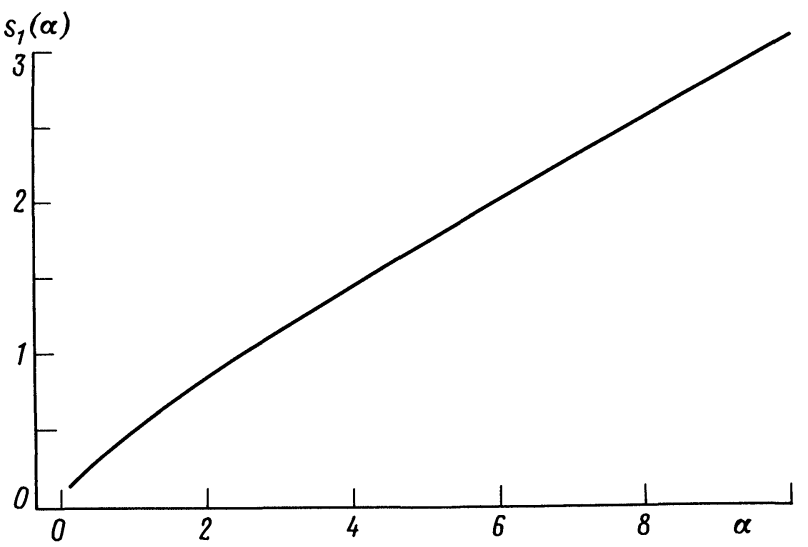

FIG. 5 The graph $s_{1}(\alpha)\left(=\frac{1}{4} \gamma^{2}(\alpha)\right)$ of the root of the equation $g_{*}(s)=0$ for $c=1$ as a function of $\alpha \geq 0$. (The computer computation was carried out by John Overdeck, AT\&T Bell Laboratories.)

where

$$
\gamma(\alpha)=2 \sqrt{s_{1}(\alpha)}
$$

It is of great interest be able to find the function $\gamma=\gamma(\alpha)$ or, at least, to study its properties.

It is clear from the above-mentioned considerations that $\gamma(0)=0, \gamma(1)=\sqrt{2}$. The numerical calculation shows us that $\gamma(3)=\sqrt{4.624 \ldots}$ (see Fig. 5).

The following consideration the idea for which belongs to M. Yor shows that for large $\alpha, \gamma(\alpha)$ behaves like $\sqrt{\alpha}$.

THEOREM 7. As $\alpha \uparrow \infty$,

$$
\frac{\gamma(\alpha)}{\sqrt{\alpha}} \rightarrow 1
$$

and (as corollary) $s_{1}(\alpha) / \alpha \rightarrow \frac{1}{4}$.

Proof. Let $X^{(\alpha)}=\left(X_{t}^{(\alpha)}\right) \in \operatorname{Bes}^{\alpha}(0)$,

$$
c(\alpha)=\mathbf{E} \max _{r \leq 1} X_{r}^{(\alpha)}
$$

It is clear from (6.34) that

$$
c(\alpha) \leq \gamma(\alpha) .
$$

Let $D(\alpha)$ be the optimal constant in the Burkholder inequality (see [9]) for the Bessel processes of order $\alpha$ :

$$
\mathbf{E} \max _{r \leq \tau} X_{r}^{(\alpha)} \leq D(\alpha) \mathbf{E} \sqrt{\tau}
$$

We shall show that

$$
\gamma(\alpha) \leq D(\alpha) .
$$

From the problem of optimal stopping considered above it follows (see (6.8)) that (at $c=1, x=0, s=0)$

$$
\sup _{\tau} \mathbf{E}\left[\max _{r \leq \tau} X_{r}^{(\alpha)}-\tau\right]=\mathbf{E}\left[\max _{r \leq \tau_{*}(1)} X_{r}^{(\alpha)}-\tau_{*}(1)\right]=s_{1}(\alpha)
$$


where $\tau_{*}(1)$ is the optimal stopping time, given by Theorem 1 . Therefore, $\mathbf{E} \max _{r \leq \tau_{*}(1)} X_{r}^{(\alpha)}=\mathbf{E} \tau_{*}(1)+s_{1}(\alpha)$ and, consequently, according to (6.39), $\mathbf{E} \tau_{*}(1)+$ $s_{1}(\alpha) \leq D(\alpha) \mathbf{E} \sqrt{\tau_{*}(1)}$. Hence,

$$
\begin{aligned}
\gamma(\alpha) & =2 \sqrt{s_{1}(\alpha)}=\inf _{T>0}\left[\sqrt{T}+\frac{s_{1}(\alpha)}{\sqrt{T}}\right] \leq \sqrt{\mathbf{E} \tau_{*}(1)}+\frac{s_{1}(\alpha)}{\sqrt{\mathbf{E} \tau_{*}(1)}} \\
& \leq D(\alpha) \frac{\mathbf{E} \sqrt{\tau_{*}(1)}}{\sqrt{\mathbf{E} \tau_{*}(1)}} \leq D(\alpha)
\end{aligned}
$$

which proves $(6.40)$.

Thus,

$$
c(\alpha) \leq \gamma(\alpha) \leq D(\alpha)
$$

It is shown in [9] that, for each stopping time $\tau$,

$$
\mathbf{E} \max _{t \leq \tau}\left|\frac{X_{t}^{(\alpha)}}{\sqrt{\alpha}}-\sqrt{t}\right| \leq C_{1} \frac{\mathbf{E} \sqrt{\tau}}{\sqrt{\alpha}}
$$

where $C_{1}$ is some absolute constant (independent of $\alpha$ and the properties of $\tau$ ). Since

$$
\left|\frac{\max _{t \leq \tau} X_{t}^{(\alpha)}}{\sqrt{\alpha}}-\sqrt{\tau}\right| \leq \max _{t \leq \tau}\left|\frac{X_{t}^{(\alpha)}}{\sqrt{\alpha}}-\sqrt{t}\right|
$$

it follows from (6.42) that, if $0<\mathbf{E} \sqrt{\tau}<\infty$, then

$$
\lim _{\alpha \rightarrow \infty} \frac{\mathbf{E} \max _{t \leq \tau}\left(X_{t}^{\alpha} / \sqrt{\alpha}\right)}{\mathbf{E} \sqrt{\tau}}=1
$$

This and (6.37) clearly show that $\lim _{\alpha \rightarrow \infty}(c(\alpha) / \sqrt{\alpha})=1$. Further, the optimal constant $D(\alpha)$ in (6.39) can be defined in the following way:

$$
D(\alpha)=\sup \frac{\mathbf{E} \max _{r \leq \tau} X_{r}^{(\alpha)}}{\mathbf{E} \sqrt{\tau}}
$$

where sup is taken over all Markov times $\tau$ such that $0<\mathbf{E} \sqrt{\tau}<\infty$. Hence,

$$
\frac{D(\alpha)}{\sqrt{\alpha}}=\sup \frac{\mathbf{E} \max \left(X_{r}^{(\alpha)} / \sqrt{\alpha}\right)}{\mathbf{E} \sqrt{\tau}}
$$

and from (6.42) it follows (for greater detail see the derivation of inequality (1.11) in [9]) that $\lim _{\alpha \rightarrow \infty} D(\alpha) / \sqrt{\alpha}=1$. Together with $\lim _{\alpha \rightarrow \infty} c(\alpha) / \sqrt{\alpha}=1$ this proves the required result. 


\section{REFERENCES}

[1] V. E. Benes,L. A. Shepp, And H. S. Witsenhauser, Some solvable stochastic control problems, Stochastics, 4 (1980), pp. 39-83.

[2] J. Bertoln, Excursions of a $\mathrm{Bes}_{0}(d)$ and its drift term $(0<d<1)$, Probab. Theory Rel. Fields, 84 (1990), pp. 231-250.

[3] - Compliments on the Hilbert transform and the fractional derivatives of Brownian local time, J. Math. Kyoto Univ., 30 (1990), pp. 651-670.

[4] - Sur une integrale pour les processus a $\alpha$-variation borneé, The Annals of Probability, 17 (1989), pp. 1521-1531.

[5] - Les processus de Dirichlet et tant qu'Espace de Banach, Stochastics, 18 (1986), pp. $155-168$.

[6] H. Chernoff, Sequential tests for the mean of a normal distribution, in: Proc. Fourth Berkeley Symp. Math. Statist. Prob., Berkeley, Cal. Press, 1961, pp. 79-92.

[7] M. Chaleyat-Maurel AND N. El Karoui, Un problème de réflection et ses applications au temps local et aux équations differentielles stochastiques sur $R$, Temps locaux, Asterisque, 1978 , pp. $52-53$, pp. $63-88$.

[8] D. R. Cox And H. D. Miller, The Theory of Stochastic Processes, Chapman and Hall, London, 1965.

[9] B. DAvis, On stopping times for $n$ dimensional Brownian motion, The Annals of Probab., 6 (1978), pp. 651-659.

[10] L. Dubins AND G. Schwarz, A sharp inequality for sub-martingales and stopping times, Asterisque, 1988, v. 157-158, pp. 129-145.

[11] H. Föllmer, Dirichlet processes, Lecture Notes in Math., 851 (1981), pp. 476-478.

[12] M. Fukushima, Dirichlet forms and Markov Processes, North-Holland, 1980.

[13] N. IKEda And S. Watanabe, Stochastic Differential Equations and Diffusion Processes, North-Holland Publ. Co, 1981.

[14] K. Itô And H. P. McKean, Diffusion Processes and Their Sample Paths, Springer-Verlag, 1965.

[15] J. JACOD AND A. N. ShIRYAev, Limit. Theorems for Stochastic Processes, Springer, 1987.

[16] D. V. Lindley, Dynamic programming and decision theory, Appl. Statist., 10 (1961), pp. 3951.

[17] H. P. MCKean, JR., The Bessel motion and a singular integral equation, Mem. College Sci. Univ., Kyoto, Ser. A. Math., 33 (1960), pp. 317-322.

[18] - A free boundary problem for the heat equation arising from a problem in mathematical economics, Industr. Manag. Rev., 6 (1965), pp. 32-39.

[19] V. S. MikhaLEvich, Bayesian choice between two hypotheses for the mean value of a normal process, Visnik Kiev Univ., 1 (1958), pp. 101-104.

[20] D. Revuz And M. Yor, Continuous Martingales and Brownian Motion, Springer-Verlag, 1991.

[21] L. C. G. Rogers AND D. Williams, Diffusions,Markov processes, and Martingales, Itô Calculus, v. 2, John Wiley and Sons, 1987.

[22] P. A. Samuelson, Rational theory of warrant pricing, Industr. Manag. Rev., 6 (1965), pp. 13-31.

[23] T. Shida and S. Watanabe, Bessel diffusions as a one-parameter family of diffusion processes, Zeitschrift für Wahr., 27 (1973), pp. 37-46.

[24] M. YoR, Sur la tranformée de Hilbert des temps locaux Browniens et une extension de la formule d'Itô, Springer-Verlag, Seminar de Probabilites XVI, Lecture Notes in Math., 920 (1982), pp. 238-247.

[25] - Some aspects of Brownian motion. I, Prepublication No $104 \mathrm{du}$ Lab. de Probab. de Univ. Paris VI, 1992.

[26] - Some aspects of Brownian motion. II, Prepublication No $125 \mathrm{du}$ Lab. de Probab. de Univ. Paris VI, 1992.

[27] B. I. Grigelionis ANd A. N. Shiryaev, On the Stefan problem and optimal stopping rules for Markov processes, Theory Probab. Appl., 11 (1966), pp. 541-558.

[28] E. B. Dynkin, The optimum choice of the time for stopping a Markov process, Doklady Akad. Nauk SSSR, 150 (1963), pp. 238-240.

[29] —, Markov Processes, Springer-Verlag, 1965.

[30] E. KAMkE, Differentialgleichungen, Chelsea, New York, 1948.

[31] R. Liptser AND A. Shiryaev, Theory of Martingales, Kluwer Acad. Publ., 1989.

[32] A. V. SKOROKHOD, Stochastic equations for diffusion processes in a bounded region. I, II. Theory Probab. Appl., 6 (1961), pp. 264-274; 7 (1962), pp. 3-23.

[33] A. N. ShIRYAev, Optimal Stopping Rules, Springer-Verlag, 1978. 